\title{
CONVEX INTEGRATION CONSTRUCTIONS IN HYDRODYNAMICS
}

\author{
TRISTAN BUCKMASTER AND VLAD VICOL
}

\begin{abstract}
We review recent developments in the field of mathematical fluid dynamics which utilize techniques that go under the umbrella name convex integration. In the hydrodynamical context, these methods produce paradoxical solutions to the fluid equations which defy physical laws. These counterintuitive solutions have a number of properties that resemble predictions made by phenomenological theories of fluid turbulence. The goal of this review is to highlight some of these similarities while maintaining an emphasis on rigorous mathematical statements. We focus our attention on the construction of weak solutions for the incompressible Euler, Navier-Stokes, and magnetohydrodynamic equations which violate these systems' physical energy laws.
\end{abstract}

\section{INTRODUCTION}

We experience turbulent fluids on a day-to-day basis. The plume rising from a lit candle starts off as smooth and well organized (laminar) and quickly becomes wildly irregular, or chaotic. The air flow around a car in motion is typically laminar around the front of the car and becomes chaotic (turbulent) in the wake of the car. Hydrodynamic turbulence has received a tremendous amount of attention over the past century, both within the physics and mathematics literature. This has resulted in a number of phenomenological theories 1 which have been very successful in making predictions about the statistics of turbulent flows. Nonetheless, to date, we do not have an unconditional, mathematically rigorous bridge between these phenomenological theories and properties of the solutions to the underlying partial differential equations (PDEs) which are meant to describe the fluid: the NavierStokes equations and their infinite Reynolds number limit, the Euler equations.

A slightly less ambitious goal, which nonetheless would offer tremendous insight into the nature of turbulent flows, is to start from experimental facts 2 translate

Received by the editors August 25, 2020.

2020 Mathematics Subject Classification. Primary 35Q35.

The first author was supported by the NSF grant DMS-1900149 and a Simons Foundation Mathematical and Physical Sciences Collaborative Grant.

The second author was supported by the NSF grant CAREER DMS-1911413.

${ }^{1}$ Some of these phenomenological theories may be traced back to the works of O. Reynolds, L. Prandtl, T. von Karman, G. I. Taylor, L. F. Richardson, W. Heisenberg, A. Kolmogorov, A. Obhukov, L. Onsager, L. Landau, E. Hopf, G. Batchelor, R. H. Kraichnan or P. G. Saffman, and many others. The topic is too vast to review here, and we refer the reader to $0,5,53,74,82,133$ for further references.

${ }^{2} \mathrm{By}$ this we mean quantitative predictions about hydrodynamic turbulence, which are confirmed experimentally both in a laboratory setting and in computer simulations to the point that these predictions are undoubted in the physics community. Examples of such experimental facts include the anomalous dissipation of energy in the infinite Reynolds number limit [158 172 or 
them into mathematical properties for solutions of the fundamental fluids PDEs, and then prove that there exist classes of solutions to these PDEs which exhibit the desired properties. In this process a certain degree of mathematical idealization is inevitable, and thus one should view such a program as showing that the PDE models are consistent with the physical reality of turbulent flows, justifying their predictive usage in computer simulations.

The remarkable outcome of such attempts is that sometimes beautiful and deep mathematical connections are revealed when trying to translate experimental facts into rigorous questions about PDEs. This article is about one such program, which has brought the method of convex integration (which has its roots in classical problems in geometry) into the hydrodynamic context, where it played the key role in the resolution of Onsager's conjecture [20,93, the mathematical manifestation of an experimental fact in turbulence.

Building upon the seminal works of Scheffer [147] and Shnirelman 152,153, De Lellis and Székelyhidi Jr. developed in [55,58, a systematic framework for applying convex integration in fluid dynamics. Since then, the mathematical techniques have evolved, in part by taking into account more detailed physical properties of the fluids equations, and this mathematical evolution has been the subject of a number of review papers $[22,57,60,61,162$. The goal of this article is to put in perspective some of the more recent results in this program, such as the nonuniqueness of distributional solutions to the Navier-Stokes equations [16, 23], and the existence of weak solutions to the ideal magneto-hydrodynamic (MHD) equations which do not conserve magnetic helicity [10].

We discuss the three flavors of convex integration encountered in hydrodynamics, which originated in the works [55], [58], and [23], respectively. The first is the classical $L_{t, x}^{\infty}$ convex integration in which one relaxes the equation, constructs a suitable notion of subsolution, to which one then adds high frequency plane wave corrections of suitable amplitudes. Through a powerful abstract functional analytic machinery originating in the field of differential inclusions, this procedure may be shown to produce a sequence of approximate solutions which converge weak-* to a bounded weak solution. The second flavor is the $C_{t, x}^{\alpha}$ convex integration scheme, which is both motivated and also resembles the earlier schemes of Nash and Kuiper for the isometric embedding problem. In this scheme, the approximate solutions are built by incrementally adding oscillatory perturbations of higher and higher frequencies. The oscillations themselves to leading order are exact stationary solutions of the underlying PDEs. The convergence to a limiting continuous weak solution holds because the error converges to zero in the uniform norm, and the approximating sequence converges absolutely in Hölder spaces. The third flavor of convex integration, intermittent convex integration, builds on the aforementioned Nash-type scheme, but the analysis is performed in Lebesgue spaces. This scheme explores the fact that if the building blocks are to leading order exact solutions of the underlying PDEs, then some of the most dangerous error terms in the iteration are linear in terms of the highest frequency perturbation, and thus via a decoupling argument these terms are smaller than initially expected when measured in the correct Lebesgue space. In this intermittent convex integration scheme, the

Kolmogorov's 4/5 law 82,159. In contrast, quantifying the intermittent nature of fully developed turbulent flows, for instance by measuring the scaling of the $p$ th-order structure functions for $p \neq 3$, is well known to exhibit variations depending on the experimental setup [82, 157]. 
cancellation of errors of smaller frequency 3 is achieved in an average sense, rather than pointwise, as is the case for the $L_{t, x}^{\infty}$ and $C_{t, x}^{\alpha}$ variants of convex integration. One way to emphasize the different types of results that may be obtained via these three approaches, is to consider hydrodynamic PDEs with more than one conservation law such as the ideal MHD equations. For this system, it is easy to discern the type of results which may be obtained via the the various flavors of convex integration [10,78, as they relate to the conservation laws of the model.

It is fair to say that the limitations of convex integrations schemes in fluid dynamics are not yet known, emphasizing the power and the flexibility of the machinery that De Lellis and Székelyhidi have developed. A large number of physically motivated, mathematically very interesting challenges remain to be explored, and we have mentioned a number of open problems and conjectures in Section 3.6. Section 4.4 and Section 5.3 .

\section{ENERGY EQUALITIES AND THEIR VALIDITY}

In this section, we discuss the energy balance relations for the Navier-Stokes, Euler, and MHD equations, which are the fundamental models governing the motion of incompressible homogenous viscous, inviscid, magnetically conducting fluids, respectively. Throughout this survey, we focus on the space dimension three (3D), on occasion making reference to two dimensional (2D) models. The physical domain in which the hydrodynamical models are considered is the periodic box $\mathbb{T}^{3}$, putting aside the many interesting physical phenomena and the mathematical difficulties which arise when considering these models in domains with solid walls. For all PDEs considered, the initial data, the forcing terms, and thus solutions satisfy periodic boundary conditions, have zero mean on $\mathbb{T}^{3}$, and are incompressible.

2.1. The Navier-Stokes equations and the zeroth law of turbulence. The PDEs governing the motion of homogenous incompressible viscous fluid flows are the Navier-Stokes equations, a manifestation of Newton's second law of motion and the conservation of mass. The unknowns are the velocity $v: \mathbb{T}^{3} \times \mathbb{R} \rightarrow \mathbb{R}^{3}$ and pressure $p: \mathbb{T}^{3} \times \mathbb{R} \rightarrow \mathbb{R}$, satisfying

$$
\begin{aligned}
\partial_{t} v+(v \cdot \nabla) v+\nabla p & =\nu \Delta v+f, \\
\operatorname{div} v & =0 .
\end{aligned}
$$

Here $\nu>0$ is the kinematic viscosity of the fluid and $f$ is a zero mean incompressible forcing term. We abuse notation and write $\nu^{-1}$ for the Reynolds number. The system (2.1) is supplemented with an initial datum $v_{0}$ which has zero mean, is incompressible, and is square integrable. Note that one may rewrite the nonlinear term in (2.1a) in divergence form as

$$
(v \cdot \nabla) v=\operatorname{div}(v \otimes v),
$$

which is important for defining distributional solutions to the system (see Definitions 2.1 and 2.2 below). The literature concerning these equations is vast, and we

\footnotetext{
${ }^{3}$ The cancellation of the previous error (Reynolds stress) in convex integration schemes essentially happens through an infinite range backwards cascade, very much like how $2 \cos ^{2}(n x)=$ $1+\cos (2 n x)$ produces a contribution of amplitude 1 at frequency 0 , no matter how large $n$ is! The weak solutions constructed via convex integration schemes essentially retain the memory of an $\mathcal{O}(1)$ access to frequency infinity. In contrast, it is known that in 3D turbulent flows, kinetic energy mostly travels from low to high frequencies, and that the energy transfer is very local, due to (2.12) below.
} 
refer the interested reader to the books $[42,67,81,114,143,170,171$ for an overview of the field.

2.1.1. The energy balance and weak solutions. Fix $\nu>0$, an initial datum $v_{0}$, and a forcing term $f$ which are smooth. Consider a smooth solution $v \in C_{t}^{1} C_{x}^{2}$ of the Navier-Stokes system (2.1) with this datum, and take an inner product of the forced momentum equation (2.1a) with $v$. Since $v$ is smooth, we obtain the pointwise energy balance

$$
\partial_{t} \frac{|v|^{2}}{2}+\operatorname{div}\left(v\left(\frac{|v|^{2}}{2}+p\right)-\nu \nabla \frac{|v|^{2}}{2}\right)=f \cdot v-\nu|\nabla v|^{2} .
$$

Integrating (2.2) over $\mathbb{T}^{3}$ and using that the divergence of a smooth periodic function vanishes, we obtain the kinetic energy balance

$$
\frac{d}{d t} \mathcal{E}+\nu \int_{\mathbb{T}^{3}}|\nabla v|^{2}=\int_{\mathbb{T}^{3}} f \cdot v
$$

where we have denoted the kinetic energy by

$$
\mathcal{E}(t)=\frac{1}{2} \int_{\mathbb{T}^{3}}|v(\cdot, t)|^{2} .
$$

Note that the second term on the left side of (2.3) is signed, and it physically represents the energy dissipation rate; the term on the right side denotes the total work of the force. We emphasize that in deriving (2.3) the following cancellation played a key role:

$$
\int_{\mathbb{T}^{3}} v \cdot((v \cdot \nabla) v+\nabla p)=\int_{\mathbb{T}^{3}} v \cdot \nabla\left(\frac{|v|^{2}}{2}+p\right)=-\int_{\mathbb{T}^{3}} \underbrace{\operatorname{div} v}_{\equiv 0}\left(\frac{|v|^{2}}{2}+p\right)=0 .
$$

For $f \in L_{t}^{2} \dot{H}_{x}^{-1}$, the energy balance (2.3) implies that

$$
\mathcal{E}(t)+\frac{\nu}{2} \int_{s}^{t}\|\nabla v\|_{L_{x}^{2}}^{2} \leq \mathcal{E}(s)+\frac{1}{2 \nu} \int_{s}^{t}\|f\|_{\dot{H}_{x}^{-1}}^{2}
$$

for any $t \geq s \geq 0$. The inequality (2.5) is the only known coercive a priori estimate for the 3D Navier-Stokes equations, and it gives an a priori bound for the solution $v$ in the so-called energy space $L_{t}^{\infty} L_{x}^{2} \cap L_{t}^{2} \dot{H}_{x}^{1}$, solely in terms of the input $v_{0} \in$ $L^{2}$ and $f \in L_{t}^{2} \dot{H}_{x}^{-1}$. We emphasize however that the knowledge that $v$ lies in $L_{t}^{\infty} L_{x}^{2} \cap L_{t}^{2} \dot{H}_{x}^{1}$ is not sufficient to establish that the cancellation relation (2.4) holds (weakly in time). This point is important to keep in mind, and it will be revisited in Section 2.1.2 below.

Using (2.5), along a sequence of approximate solutions for which (2.3) is justified, Leray [115] (and later Hopf [89] in the case of domains with boundary) proved that for any finite energy initial datum there exists a global weak solution to the NavierStokes equation. More precisely, Leray proved the global existence in the following class of weak solutions.

Definition 2.1 (Leray-Hopf weak solution). A vector field

$$
v \in C_{\text {weak }}^{0}\left([0, \infty) ; L^{2}\left(\mathbb{T}^{3}\right)\right) \cap L^{2}\left([0, \infty) ; \dot{H}^{1}\left(\mathbb{T}^{3}\right)\right)
$$

is called a Leray-Hopf weak solution of the Navier-Stokes equations if for any $t \in \mathbb{R}$ the vector field $v(\cdot, t)$ is weakly divergence free, has zero mean, satisfies the 
Navier-Stokes equations distributionally:

$$
\int_{\mathbb{R}} \int_{\mathbb{T}^{3}} v \cdot\left(\partial_{t} \varphi+(v \cdot \nabla) \varphi+\nu \Delta \varphi+f\right) d x d t+\int_{\mathbb{T}^{3}} v(\cdot, 0) \cdot \varphi(\cdot, 0) d x=0,
$$

for any divergence free test function $\varphi \in C_{0}^{\infty}\left([0, \infty), C^{\infty}\left(\mathbb{T}^{3}\right)\right)$, and satisfies the energy inequality (2.5) for $t \geq 04$

While Leray-Hopf weak solutions are known to enjoy certain desirable properties such weak-strong uniqueness [115, 142, 150, 176] (if a smooth solution exists, then any Leray-Hopf weak solution with the same initial datum is equal to this smooth one) or epochs of regularity [115] (intervals for which the solution is smooth, leading to partial regularity in time [111,144,145]), their uniqueness to date remains open. Regularity, and hence also uniqueness, is only known to hold under the extra assumption that the solution is bounded in a scaling invariant space, such as $L_{t}^{p} L_{x}^{q}$ for $2 / p+3 / q=1$, known as the Ladyženskaja-Prodi-Serrin conditions [72,103, 142, 149]. In fact, it has been conjectured [97, 98, 112 that the uniqueness of Leray-Hopf weak solutions fails as soon as the Ladyženskaja-Prodi-Serrin conditions are violated. In spite of compelling numerical evidence [87, to date this question remains open.

Note that the distributional form of the Navier-Stokes equations (2.6) makes sense as soon as $v \in L_{t}^{2} L_{x}^{2}$, without requiring that $v \in L_{t}^{2} \dot{H}_{x}^{1}$. Thus, as in [150] one may define an even weaker notion of solution to (2.1) as follows.

Definition 2.2 (Weak/mild solution). A vector field $v \in C_{\text {weak }}^{0}\left([0, \infty) ; L^{2}\left(\mathbb{T}^{3}\right)\right)$ is called a weak or distributional solution of the Navier-Stokes equations if for any $t$ the vector field $v(\cdot, t)$ is weakly divergence free, has zero mean, and (2.6) holds for for any smooth divergence free test function $\varphi$.

As shown in [75, the weak solutions of Definition 2.2 satisfy the integral equation

$$
v(\cdot, t)=e^{\nu \Delta t} v(\cdot, 0)+\int_{0}^{t} e^{\nu \Delta(t-s)} \mathbb{P} \operatorname{div}(v(\cdot, s) \otimes v(\cdot, s)) d s,
$$

and are called mild solutions [114, Definition 6.5]; here $\mathbb{P}$ is the Helmholtz projection. We emphasize that this class of solutions is weaker than the Leray-Hopf weak solutions in Definition 2.1 because solutions need not satisfy the energy inequality (2.5), and their energy dissipation rate need not be finite. Similarly to Leray-Hopf ones, these weak solutions are known to be regular under the additional assumption that one of the Ladyženskaja-Prodi-Serrin conditions is satisfied $75,84,85,109,113,120$. However, as opposed to Leray-Hopf weak solutions, for the weak solutions of Definition 2.2 it is by now known that uniqueness fails. This was established by the authors of this review in [23] (see Theorem 4.1 below) and then improved in a joint work with Colombo [16] showing that uniqueness fails even if partial regularity in time holds (see Theorem 4.3 below). The proofs in [16, 23. rely on a version of the convex integration scheme, called intermittent convex integration, which will be discussed in Section 4 below.

\footnotetext{
${ }^{4}$ A stronger form of the energy inequality holds by Leray's construction: 2.5 holds for a.e. $s \geq 0$ and all $t>s$. Moreover, an a priori stronger form of weak solution may be constructed by modifying Leray's proof. These so-called suitable weak solutions obey a version of the energy inequality which is localized in space and time, which allows one to prove that they are smooth except for a putative singular space-time set with one-dimensional (1D) parabolic Hausdorff measure which is zero [25 145 146]; see also the more recent reviews 114, 140, 143.
} 
2.1.2. Conditional proof of energy balance. We return to a question alluded to earlier: Consider a weak/mild solution of the Navier-Stokes equations which is known to lie in the Leray-Hopf energy space $L_{t}^{\infty} L_{x}^{2} \cap L_{t}^{2} \dot{H}_{x}^{1}$. Does such a solution automatically obey the energy equality/balance (2.3)? Currently, this question is open and only conditional criteria are available. The issue lies in justifying the cancellation (2.4) in the sense of temporal distributions. The classical results state that if in addition to $v \in L_{t}^{\infty} L_{x}^{2} \cap L_{t}^{2} \dot{H}_{x}^{1}$ one also knows that $v \in L_{t}^{4} L_{x}^{4}$ [118] or, more generally, $v \in L_{t}^{p} L_{x}^{q}$ with $2 / p+2 / q=1$ [151, then (2.3) holds; see also [32, 33, 110, 116] for more recent refinements. We emphasize that these conditional results assume less integrability on $v$ than the Ladyženskaja-Prodi-Serrin conditions, and this is a common theme in hydrodynamics: the energy equality may be justified under much weaker conditions than those required for establishing the uniqueness of solutions, i.e., the energy equality is a weaker notion of rigidity than uniqueness [104].

We recall a modern proof of the classical result of 118, 151, namely that $v \in$ $L_{t}^{4} L_{x}^{4} \cap C_{\text {weak }, t}^{0} L_{x}^{2} \cap L_{t}^{2} \dot{H}_{x}^{1}$ implies (2.3); for details we refer the reader to [29, 33, 154]. The point is that by interpolation, the assumption on $v$ implies that $v \in L_{t}^{3} B_{3, c_{0}, x}^{1 / 3}$, which means that $\lim _{|z| \rightarrow 0} \frac{1}{|z|} \int_{0}^{T} \int_{\mathbb{T}^{3}}|v(x+z, t)-v(x, t)|^{3} d x d t=0$. The former condition is known due to [29,68] to imply (2.3). The more detailed argument is as follows.

Denote by $\mathbb{P}_{\leq \kappa} v$ the projection of $v$ to its Fourier frequencies which have modulus less than $\kappa$. Testing the Navier-Stokes equations with the smooth divergence free function $\mathbb{P}_{\leq \kappa} v=\mathbb{P}_{<\kappa}^{2} v$, and using that $\mathbb{P}_{\leq \kappa}$ is self-adjoint on $L_{x}^{2}$ and commutes with space and time derivatives, similarly to (2.3) we obtain that

$$
\frac{d}{d t} \int_{\mathbb{T}^{3}} \frac{\left|\mathbb{P}_{\leq \kappa} v\right|^{2}}{2} d x+\nu \int_{\mathbb{T}^{3}}\left|\nabla \mathbb{P}_{\leq \kappa} v\right|^{2}=\int_{\mathbb{T}^{3}} \mathbb{P}_{\leq \kappa} f \cdot \mathbb{P}_{\leq \kappa} v d x+\Pi_{\kappa},
$$

where we have denoted by $\Pi_{\kappa}$ the energy flux through frequencies $\approx \kappa$ by, i.e.,

$$
\Pi_{\kappa}=\int_{\mathbb{T}^{3}} \mathbb{P}_{\leq \kappa}(v \otimes v): \nabla \mathbb{P}_{\leq \kappa} v .
$$

First, we note that as soon as $v \in C_{\text {weak }, t}^{0} L_{x}^{2} \cap L_{t}^{2} \dot{H}_{x}^{1}$, by the dominated convergence theorem, the terms on the left side of (2.7) converge as $\kappa \rightarrow \infty$ to the terms on the left side of (2.3), when integrated in time. Second, if $f \in L_{t}^{2} \dot{H}_{x}^{-1}$, then also the first term on the right side of (2.7) converges to the term on the right side of (2.3), when integrated in time.

The only question that remains is whether the time integrated flux term in (2.7), $\int_{0}^{T} \Pi_{\kappa} d t$, vanishes as $\kappa \rightarrow \infty$. In order to address this, one first uses that $\mathbb{P}_{\leq \kappa} v$ is smooth, and thus the cancellation (2.4) holds with $v$ replaced by $\mathbb{P}_{\leq \kappa} v$. This allows one to rewrite

$$
\Pi_{\kappa}(v)=\int_{\mathbb{T}^{3}}\left(\mathbb{P}_{\leq \kappa}(v \otimes v)-\left(\mathbb{P}_{\leq \kappa} v \otimes \mathbb{P}_{\leq \kappa} v\right)\right): \nabla \mathbb{P}_{\leq \kappa} v=\int_{\mathbb{T}^{3}} \mathcal{C}_{\kappa}(v, v): \nabla \mathbb{P}_{\leq \kappa} v,
$$

which reveals the importance of the quadratic commutator term

$$
\mathcal{C}_{\kappa}(v, w)=: \mathbb{P}_{\leq \kappa}(v \otimes w)-\mathbb{P}_{\leq \kappa} v \otimes \mathbb{P}_{\leq \kappa} w
$$

As was shown by Constantin, E, and Titi in [41, we have

$$
\left\|\mathcal{C}_{\kappa}(v, w)\right\|_{L_{x}^{3 / 2}} \lesssim \kappa^{-2 \alpha}\|v\|_{B_{3, \infty, x}^{\alpha}}\|w\|_{B_{3, \infty, x}^{\alpha}} .
$$


This may be combined with the bound $\left\|\nabla \mathbb{P}_{\leq \kappa} v\right\|_{L_{x}^{3}} \lesssim \kappa^{1-\alpha}\|v\|_{B_{3, \infty}^{\alpha}, x}$ to yield

$$
\left|\Pi_{\kappa}(v)\right| \lesssim \kappa^{1-3 \alpha}\|v\|_{B_{3, \infty, x}^{\alpha}}^{3},
$$

which proves that as soon as $v \in L_{t}^{3} B_{3, \infty, x}^{\alpha}$ for any $\alpha>1 / 3$, then $\Pi_{\kappa} \rightarrow 0$ as $\kappa \rightarrow \infty$, thereby proving that the energy equality holds. In fact, this is exactly the proof given in [41] for the rigid side of Onsager's conjecture; we discuss this in Section 2.2 below.

Returning to our goal of proving the energy equality for the Navier-Stokes system (2.3), we note that the assumption $v \in L_{t}^{4} L_{x}^{4}$ was not yet used. Using standard interpolation inequalities, the information that $v \in L_{t}^{4} L_{x}^{4} \cap C_{\text {weak }, t}^{0} L_{x}^{2} \cap L_{t}^{2} \dot{H}_{x}^{1}$ gives that $v \in L_{t}^{3} W_{x}^{1 / 3,3}$ and thus that $v \in L_{t}^{3} B_{3, \infty, x}^{1 / 3}$ and $\lim _{i \rightarrow \infty} 2^{i}\left\|\mathbb{P}_{\approx 2^{i}} v\right\|_{L_{x, t}^{3}}^{3}=0$, where $\mathbb{P}_{\approx 2^{i}}=\mathbb{P}_{\leq 2^{i+1}} \mathbb{P}_{\geq 2^{i-1}}$; i.e., $v$ lies in the space $L_{t}^{3} B_{3, c_{0}, x}^{1 / 3}$. This information is not good enough to apply (2.11) since $\alpha=1 / 3$; it is however just good enough to prove that the flux vanishes as $\kappa \rightarrow \infty$. To see this, we recall a more detailed estimate on the commutator term $\mathcal{C}_{\kappa}(v, v)$, and thus for the flux $\Pi_{\kappa}$, which was obtained in [29] by using the Bony paraproduct decomposition from Littlewood-Paley analysis:

$$
\left|\Pi_{2^{j}}(v)\right| \lesssim \sum_{i=1}^{\infty} 2^{-2 / 3|j-i|} 2^{i}\left\|\mathbb{P}_{\approx 2^{i}} v\right\|_{L^{3}}^{3} .
$$

Besides showing that the energy transfer from one dyadic scale to another is mainly local, the above estimate shows that $v \in L_{t}^{3} B_{3, c_{0}, x}^{1 / 3}$ implies $\lim _{\kappa \rightarrow \infty} \int_{0}^{T} \Pi_{\kappa}=0$, thereby completing the conditional proof of (2.3). We note that estimate (2.12) gives the best-known condition on $v$ which ensures that the total energy flux vanishes, and this condition is sharp in the case of the 1D Burgers equation [154]. We revisit these ideas in Section 2.2 .

2.1.3. Anomalous dissipation of energy in the infinite Reynolds number limit. We have seen in the previous section that the energy equality (2.3) is not necessarily satisfied for weak solutions of the Navier-Stokes equations, even if they lie in the Leray-Hopf space. The identity (2.7) does however show upon passing $\kappa \rightarrow \infty$ that

$$
\frac{d}{d t} \mathcal{E}=\int_{\mathbb{T}^{3}} f \cdot v+\Pi_{\infty}(v)-\nu \int_{\mathbb{T}^{3}}|\nabla v|^{2}
$$

holds in the sense of distributions in time, and $\Pi_{\infty}(v)=\lim _{\kappa \rightarrow \infty} \Pi_{\kappa}(v)$ may or may not be equal to 0 . It is thus natural to define the average energy dissipation rate on the time interval $[0, T]$ as

$$
\varepsilon_{\nu, T}:=\nu f_{0}^{T} \int_{\mathbb{T}^{3}}|\nabla v|^{2}-f_{0}^{T} \Pi_{\infty}(v) .
$$

It is clear from the above definition that if the Navier-Stokes solutions maintain a certain degree of regularity uniformly in $\nu \ll 1$, for instance if at fixed $\nu>0$ we have $v \in L_{t}^{4} L_{x}^{4}$ and if $\nu\|v\|_{L_{t}^{2} \dot{H}_{x}^{1}}^{2} \rightarrow 0$ when $\nu \rightarrow 0$, then for any $T>0$ we have $\lim _{\nu \rightarrow 0} \varepsilon_{\nu, T}=0$.

The vanishing of the energy dissipation rate for turbulent solutions as $\nu \rightarrow 0$ (equivalently, as the Reynolds number goes to $\infty$ ) contradicts an experimental fact 
known as the zeroth law of turbulence, which loosely speaking states that 5

$$
\liminf _{\nu \rightarrow 0} \varepsilon_{\nu, \infty}=\varepsilon>0,
$$

for an $\varepsilon \in(0, \infty)$. The anomalous dissipation of energy postulated in (2.15) is the fundamental ansatz of Kolmogorov's 1941 theory of fully developed turbulence [105 107], and it has been verified experimentally to a tremendous accuracy 99, 141, 158. The literature on this topic is vast, and we refer the interested reader to $22,74,82,154$ for references.

To date it remains open to prove that (2.15) is a manifestation of the statistical behavior of solutions to the Navier-Stokes equations in the infinite Reynolds number limit. Nonetheless, 2.15) provides unquestionable physical evidence that in the limit $\nu \rightarrow 0$, one should expect that Navier-Stokes solutions do not remain uniformly smooth, and that (at best) they converge to nonsmooth, possibly nonunique, weak solutions of the Euler equations. Thus, in an attempt to translate predictions made by turbulence theories into mathematically rigorous questions, such as Onsager's conjecture which we discuss next, it is natural to work within the framework of weak solutions of the Euler equations.

2.2. The Euler equations and Onsager's conjecture. The classical model for the motion of an incompressible homogenous inviscid fluid are the Euler equations, obtained by formally letting $\nu=0$ in (2.1)

$$
\begin{aligned}
\partial_{t} v+(v \cdot \nabla) v+\nabla p & =0, \\
\operatorname{div} v & =0 .
\end{aligned}
$$

As with the Navier-Stokes equations, the literature concerning the incompressible Euler equations is immense, and we refer the interested reader to the books 28 , 119, 124, 125] for an overview of the standard results in the field.

2.2.1. The energy equality and weak solutions. In direct correspondence to the discussion presented in Section 2.1.1 for the Navier-Stokes equations, one may show that smooth solutions $v \in C_{t}^{1} C_{x}^{1}$ of the Euler equations conserve their kinetic energy

$$
\frac{d}{d t} \mathcal{E}=0 \text {. }
$$

Indeed, (2.17) is nothing but (2.3) with $\nu=0$ and $f=0$. Thus, any reasonable notion of solution to the Euler equations should at least have a finite $L_{t}^{\infty} L_{x}^{2}$ norm.

On the other hand, as mentioned in Section 2.1.3 the zeroth law of turbulence motivates the study of weak solutions to the Euler equations, defined as:

Definition 2.3 (Weak solution). A vector field $v \in L^{\infty}\left(I ; L^{2}\left(\mathbb{T}^{3}\right)\right)$ is called a weak solution of the Euler equations on an open $I \subset \mathbb{R}$ if for any $t \in I$ the vector field $v(\cdot, t)$ is weakly divergence free, has zero mean, and $v$ satisfies (2.16) in the sense of distributions; that is,

$$
\int_{\mathbb{R}} \int_{\mathbb{T}^{3}} v \cdot\left(\partial_{t} \varphi+(v \cdot \nabla) \varphi\right) d x d t=0
$$

\footnotetext{
${ }^{5}$ Here, as in laboratory experiments, we consider the long (infinite) time average of the energy dissipation rate, $\varepsilon_{\nu, \infty}$, to signify that the solution has reached a stationary regime. For the sake of brevity we avoid the important and subtle discussion about ensemble averages with respect to probability measures on $L^{2}$ that encode the statistics of the flow 7980 , and about the impromptu ergodic hypothesis which is classical in statistical mechanics. We refer the interested reader to 82 ] or other texts in turbulence.
} 
holds for any divergence free test function $\varphi \in C_{0}^{\infty}\left(I ; C^{\infty}\left(\mathbb{T}^{3}\right)\right)$. The pressure can be recovered by the formula $-\Delta p=\operatorname{div} \operatorname{div}(v \otimes v)$ with $p$ of zero mean.

2.2.2. Onsager's conjecture and its variants. The validity of (2.17) for weak (instead of smooth) solutions of the Euler equations is the subject of Onsager's conjecture, one of the most celebrated connections between phenomenologies in turbulence and the rigorous mathematical analysis of PDEs arising in fluid dynamics. In [138, Onsager considered the possibility that energy dissipation in the infinite Reynolds number limit is not caused by a remnant of viscous effects (i.e., from the term $-\nu\|\nabla v\|_{L^{2}}^{2}$ present on the right side of $(\underline{2.13})$ ), but instead, because the solutions of the limiting equation at $\nu=0$, namely the Euler equation, are not sufficiently smooth to ensure that $\Pi_{\infty}=0$. As explained in [73], the argument that the energy equality (2.17) holds for a finite energy solution $v$ of the Euler equations if and only if the total energy flux vanishes (i.e., $\lim _{\kappa \rightarrow \infty} \Pi_{\kappa}(v)=0$ when time integrated) is essentially already contained in [138]. Onsager's remarkable analysis (see also [74) went further and made a precise statement about the threshold regularity of $v$ which is necessary in order to justify (2.17); in modern mathematical terms Onsager's conjecture 6 is now a theorem due to [20, 41, 93]:

Theorem 2.4 (Onsager's conjecture in Hölder spaces).

(a) Any weak solution $v$ belonging to the Hölder space $C_{x, t}^{\alpha}$ for $\alpha>1 / 3$ conserves its kinetic energy.

(b) For any $\alpha<1 / 3$ there exist weak solutions $v \in C_{x, t}^{\alpha}$ which dissipate kinetic energy, i.e., the kinetic energy $\mathcal{E}(t)$ is a nonincreasing function of time.

The rigidity, part (a) of Theorem 2.4, is discussed in Section 2.2.3, while the flexibility, part (b) , is presented in Section 3 .

Remark 2.5 (Onsager's conjecture on other Banach scales). In Theorem 2.4, the threshold between rigidity and flexibility is measured here on the Hölder $C_{t, x}^{\alpha}$ scale, with threshold value $\alpha=1 / 3$. However, since the energy flux $\Pi_{\kappa}$ is trilinear in $v$ (see (2.8) ), it is most natural to measure this dichotomy on an $L^{3}$ based space, such as the scale of spaces $L_{t}^{3} B_{3, \infty, x}^{\alpha}$. The bound (2.11) again suggests that the threshold value on the $L^{3}$-based Banach scale is $\alpha=1 / 3$, and indeed the proof of Theorem 2.4 establishes this fact. Lastly, we note that the threshold between rigidity (回) and flexibility (b) may alternatively be measured on $L^{2}$ based spaces in $x$, such as the $L_{t}^{3} H_{x}^{\alpha}$, with $\alpha \geq 0$. These spaces are classically related to Fourier analytic measurements of energy spectra (such as the Kolmogorov-Obhukov 5/3 power spectrum) and to the scaling of second-order structure functions in turbulent flows [82. On this Sobolev scale, however, the value of the threshold exponent is unclear. It is known for a while [32, 160 that the kinetic energy is conserved if $v \in L_{t}^{3} H_{x}^{\alpha}$ with $\alpha>5 / 6$. However, as a byproduct of the proof of the flexibility part (b) of Theorem [2.4, we only have the existence of weak solutions which violate the energy equation for $\alpha<1 / 3$. Based on physical considerations and on the experimentally measured anomalous scaling of second-order structure functions in fully developed turbulent flows [82, it is safe to conjecture that the threshold exponent on the $L_{t}^{3} H_{x}^{\alpha}$ is strictly larger than $1 / 3$; it is, however, not clear whether it

\footnotetext{
${ }^{6}$ For a discussion of Onsager's conjecture for the $2 \mathrm{D}$ Euler equations, we refer the reader to 29 30 44.
} 
should be equal to $5 / 6$ or another smaller value 7 Conservatively, we conjecture (see also Open Problem 5 in 22]) that Onsager's threshold exponent on the $L^{2}$ based Sobolev scale is strictly larger than $1 / 3$ :

Conjecture 2.6 (Deviation from the Kolmogorov-Obhukov power spectrum). There exists an $\alpha \in(1 / 3,5 / 6)$ and infinitely many weak solutions $v \in C_{t} H_{x}^{\alpha}$ of the 3D Euler equations (2.16), which dissipate kinetic energy.

2.2.3. The proof of rigidity in Onsager's conjecture. Part (a) of Onsager's conjecture was partially established by Eyink in [73] and later proven in full by Constantin, E, and Titi in [41. Indeed, the commutator bound (2.11) established in [41] shows that if $v \in L_{t}^{3} C_{x}^{\alpha}$ with $\alpha>1 / 3$, then automatically by embedding we have $v \in L_{t}^{3} B_{3, \infty, x}^{\alpha}$, and so

$$
\lim _{\kappa \rightarrow \infty} \int_{0}^{T}\left|\Pi_{\kappa}(v)\right| \lesssim \lim _{\kappa \rightarrow \infty} \kappa^{1-3 \alpha}\|v\|_{L^{3}\left(0, T ; B_{3, \infty}^{\alpha}\right)}^{3}=0,
$$

thereby proving (2.17). The argument in [41] was further refined in 68, where the energy dissipation measure was introduced, in 29] where the bound (2.12) is proven, and in the more recent paper [156], which discusses several geometric constraints which ensure the conservation of energy for the threshold value $\alpha=1 / 3$. We note that the proof of rigidity on the $L^{2}$ based scale (i.e., for $v \in L_{t}^{3} H_{x}^{\alpha}$ with $\alpha>5 / 6)$ also follows from the bound (2.12) established in [29] by additionally using the Bernstein inequality $\left\|\mathbb{P}_{\approx 2^{i}} v\right\|_{L^{3}} \lesssim 2^{\frac{i}{2}}\left\|\mathbb{P}_{\approx 2^{i}} v\right\|_{L^{2}}$.

2.2.4. Helicity. We mention that besides the kinetic energy $\mathcal{E}$, the $3 \mathrm{D}$ Euler system also posses one more nontrivial invariant [71] which is not coercive but has deep geometric meaning [131]; this is the helicity

$$
\mathcal{H}_{\omega, \omega}(t)=\int_{\mathbb{T}^{3}} v(\cdot, t) \cdot \omega(\cdot, t),
$$

where $\omega=\nabla \times v$ is the vorticity. Here we use the generalized helicity notation [4]

$$
\mathcal{H}_{f, g}=\int_{\mathbb{T}^{3}}\left(\nabla \times(-\Delta)^{-1} f\right) \cdot g d x,
$$

which will appear several times in Section 2.3. As opposed to the kinetic energy, which is well defined for weak solutions in the sense of Definition 2.3 the fluid helicity requires a minimal regularity of $v \in L_{t}^{2} \dot{H}_{x}^{1 / 2}$ to be well defined, via the duality pairing between $\dot{H}^{1 / 2}$ and $\dot{H}^{-1 / 2}$. However, it is not known whether for any weak solution $v \in L_{t}^{3} \dot{H}_{x}^{1 / 2}$ we have

$$
\frac{d}{d t} \mathcal{H}_{\omega, \omega}=0
$$

Due to [29], we know that if $v \in L_{t}^{3}\left(B_{3, c_{0}, x}^{2 / 3} \cap \dot{H}_{x}^{1 / 2}\right)$, then the fluid helicity for that weak solution is a constant function of time. In terms of rigidity, this condition is expected to be sharp. An alternative condition in which velocity and vorticity obey different assumptions was obtained in 63.

On the other hand, in terms of flexibility we note that for the weak solutions constructed for part (b) of Theorem 2.4 the helicity is not well defined, as $C^{1 / 3}$

\footnotetext{
${ }^{7}$ For a dyadic shell model of the 3D Euler equations, for which the energy flux is unidirectional, it was shown in 31] that the threshold $\alpha=5 / 6$ is sharp.
} 
does not embed into $\dot{H}^{1 / 2}$. In fact, to date the following question remains widely open:

Conjecture 2.7 (Helicity flexibility). There exists a weak solution $v$ of the 3D Euler equations (2.16), with $v \in C_{t} \dot{H}_{x}^{\alpha}$ for some $\alpha \geq 1 / 2$, such that the helicity $\mathcal{H}_{\omega, \omega}$ is not a constant function of time.

The difficulty is that on the one hand for the weak solutions obtained from the convex integration constructions in Section 3, the kinetic energy can be always made to be a nonconstant function of time; on the other hand, if one works on the Hölder scale $C^{\alpha}$ and wishes to have a well-defined helicity, then one has to take $\alpha \geq 1 / 2$; but in turn this implies that the kinetic energy must be conserved due to part (国) of Theorem 2.4. It is conceivable that in order to attack Conjecture 2.7 one has to work on the $L^{2}$ based Sobolev scale $L_{t}^{\infty} H_{x}^{\alpha}$, and that the intermittent convex integration which we will describe in Section 4 should be employed instead. Even so, Conjecture 2.7 is strictly harder than Conjecture 2.6.

2.3. The MHD equations and Taylor's conjecture. The incompressible MHD equations are the classical macroscopic model coupling Maxwell's equations to the evolution of an electrically conducting incompressible fluid [12,52]. The unknowns are the velocity field $v$, the magnetic field $B$, and the scalar pressure $p$, which we take to have zero mean on $\mathbb{T}^{3}$. The ideal (i.e., inviscid and nonresistive) version of these equations is

$$
\begin{aligned}
& \partial_{t} v+(v \cdot \nabla) v-(B \cdot \nabla) B+\nabla p=0, \\
& \partial_{t} B+(v \cdot \nabla) B-(B \cdot \nabla) v=0, \\
& \operatorname{div} v=\operatorname{div} B=0 .
\end{aligned}
$$

The viscous $(\nu>0)$ and resistive $(\mu>0)$ MHD equations are given by

$$
\begin{aligned}
& \partial_{t} v+(v \cdot \nabla) v-(B \cdot \nabla) B+\nabla p=\nu \Delta v, \\
& \partial_{t} B+(v \cdot \nabla) B-(B \cdot \nabla) v=\mu \Delta B, \\
& \operatorname{div} v=\operatorname{div} B=0 .
\end{aligned}
$$

Setting $B=0$ in (2.19) we recover the Euler equations (2.16), while letting $B=0$ in (2.20) we arrive at the Navier-Stokes equations (2.1). As such, the local-in-time theory for smooth solutions of the MHD equations, as well as the global-in-time theory for weak solutions, closely mimics the one for the Euler and Navier-Stokes systems; see [69, 148.

2.3.1. Ideal MHD conservation laws and weak solutions. The ideal MHD equations (2.19) posses a number of global invariants [101, two of which are obtained via a direct analogy with the Euler equation, namely the total energy and the cross helicity, and one more which is intrinsic to the Lie transport of the magnetic field (2.19a), namely the magnetic helicity. The fact that these conservation laws are not defined at the same level of spatial regularity, makes the analysis in a sense more challenging than for 3D Euler.

The total energy

$$
\mathcal{E}(t)=\frac{1}{2} \int_{\mathbb{T}^{3}}|v(\cdot, t)|^{2}+|B(\cdot, t)|^{2}
$$


gives the only known coercive conserved quantity (and in fact the Hamiltonian 101, 173 of the system), for smooth solutions of (2.19). In order to verify that $\mathcal{E}(t)$ is a constant function of time, it is convenient to rewrite (2.19) in terms of the Elsässer variables

$$
z_{+}=v+B \quad \text { and } \quad z_{-}=v-B,
$$

so that the system (2.19) becomes

$$
\begin{aligned}
& \partial_{t} z_{ \pm}+\left(z_{\mp} \cdot \nabla\right) z_{ \pm}+\nabla q=0, \quad q=p+\frac{|B|^{2}}{2}, \\
& \operatorname{div} z_{ \pm}=0 .
\end{aligned}
$$

Testing the momentum equation for $z_{ \pm}$with $z_{ \pm}$, integrating over $\mathbb{T}^{3}$, and using that both $z_{+}$and $z_{-}$are incompressible, similarly to (2.17) we obtain that if $v, B \in C_{t}^{1} C_{x}^{1}$ (and thus $z_{ \pm} \in C_{t}^{1} C_{x}^{1}$ ), then

$$
\frac{d}{d t} \int_{\mathbb{T}^{3}}\left|z_{+}(\cdot, t)\right|^{2}=\frac{d}{d t} \int_{\mathbb{T}^{3}}\left|z_{-}(\cdot, t)\right|^{2}=0 .
$$

On the other hand, from (2.22) we have that

$$
\mathcal{E}(t)=\frac{1}{4} \int_{\mathbb{T}^{3}}\left|z_{+}(\cdot, t)\right|^{2}+\left|z_{-}(\cdot, t)\right|^{2},
$$

which combined with (2.24) shows that $\mathcal{E}$ is conserved for smooth solutions.

Besides the total energy, the MHD system possesses one more Elsässer invariant [2], the cross helicity

$$
\mathcal{H}_{\omega, B}=\int_{\mathbb{T}^{3}} v(\cdot, t) \cdot B(\cdot, t)=\frac{1}{4} \int_{\mathbb{T}^{3}}\left|z_{+}(\cdot, t)\right|^{2}-\left|z_{-}(\cdot, t)\right|^{2} .
$$

Here and throughout the paper we use the notation in (2.18). Again, (2.24) shows that $\mathcal{H}_{\omega, B}$ is conserved for smooth solutions.

We note that both $\mathcal{E}$ and $\mathcal{H}_{\omega, B}$ are bounded functions of time as soon as $v, B \in$ $L_{t}^{\infty} L_{x}^{2}$, and in view of the positivity of the total energy, any meaningful notion of solution for (2.19) should at the very least assume this amount of regularity. This motivates the notion of weak solution for the ideal MHD system (in analogy to Definition 2.3 for Euler):

Definition 2.8 (Weak/distributional solution). We say $(v, B) \in L^{\infty}\left(I ; L^{2}\left(\mathbb{T}^{3}\right)\right)$ is a weak solution of the ideal MHD system (2.19) on an open interval $I \subset \mathbb{R}$, if for any $t \in I$, the vector fields $u(\cdot, t)$ and $B(\cdot, t)$ are divergence free in the sense of distributions, they have zero mean, and (2.19) holds in the sense of distributions; i.e.,

$$
\begin{aligned}
& \int_{\mathbb{R}} \int_{\mathbb{T}^{3}} \partial_{t} \varphi \cdot v+\nabla \varphi:(v \otimes v-B \otimes B) d x d t=0, \\
& \int_{\mathbb{R}} \int_{\mathbb{T}^{3}} \partial_{t} \varphi \cdot B+\nabla \varphi:(v \otimes B-B \otimes v) d x d t=0
\end{aligned}
$$

hold for all divergence free test functions $\varphi \in C_{0}^{\infty}\left(I ; C^{\infty}\left(\mathbb{T}^{3}\right)\right)$.

The validity of (2.24) for weak solutions of the ideal MHD system gives rise to an Onsager-type analysis, which we discuss in Section 2.3 .2 below. 
As alluded to at the beginning of the section, the ideal MHD system has one more conservation law, the magnetic helicity, which is defined as

$$
\mathcal{H}_{B, B}(t)=\int_{\mathbb{T}^{3}} A(\cdot, t) \cdot B(\cdot, t)
$$

(see 131, 177, 178), where $A$ is a vector potential for $B$, i.e., a zero mean periodic field such that curk $A=B$. As we work on the simply connected domain $\mathbb{T}^{3}$ and $B$ is incompressible, the value of $\mathcal{H}_{B, B}(t)$ is independent of the gradient part of $A$; thus $A$ may be chosen without loss of generality such that $\operatorname{div} A=0$, so that $A=\nabla \times(-\Delta)^{-1} B$. Thus, the definition (2.27) is consistent with the definition (2.18) introduced earlier.

In order to see that for smooth solutions $(v, B)$ of (2.19) we have

$$
\frac{d}{d t} \mathcal{H}_{B, B}=0
$$

one may proceed as follows. Since the Biot-Savart operator $B \mapsto A=\nabla \times(-\Delta)^{-1} B$ is self-adjoint, and since for divergence free $v$ and $B$ we may rewrite $B \cdot \nabla v-v \cdot \nabla B=$ $\nabla \times(v \times B)$, we have

$$
\begin{aligned}
\frac{d}{d t} \mathcal{H}_{B, B} & =2 \int_{\mathbb{T}^{3}}\left(\nabla \times(-\Delta)^{-1} B\right) \cdot \partial_{t} B \\
& =2 \int_{\mathbb{T}^{3}}\left(\nabla \times(-\Delta)^{-1} B\right) \cdot(\nabla \times(v \times B)) \\
& =2 \int_{\mathbb{T}^{3}} \nabla \times\left(\nabla \times(-\Delta)^{-1} B\right) \cdot(v \times B) \\
& =2 \int_{\mathbb{T}^{3}} \underbrace{B \cdot(v \times B)}_{\equiv 0}=0 .
\end{aligned}
$$

In the second to last equality we have used the identity $\nabla \times(\nabla \times B)=\nabla(\nabla \cdot B)-\Delta B$.

We emphasize that as opposed to the total energy and the cross helicity which require $(v, B) \in L_{t}^{\infty} L_{x}^{2}$ in order to be well defined, the magnetic helicity is well defined as soon as $B \in L_{t}^{\infty} \dot{H}_{x}^{-1 / 2}$, a negative level of regularity. This difference is manifested in the context of reconnection events in magneto-hydrodynamic turbulence, via a phenomenon whose mathematical aspects are described by Taylor's conjecture 11, 132, 168, 169, see Section 2.3.4 below.

Remark 2.9 (2D Euler and Surface Quasi Geostrophic). This situation encountered here in which the hydrodynamic model has conservation laws at different levels of regularity ( $\mathcal{E}$ vs $\mathcal{H}_{B, B}$ ) is not uncommon. Another occurrence is in the context of the 2D Surface Quasi Geostrophic (SQG) and 2D Euler equations. For both of these equations smooth solutions conserve the $L^{2}$ norm (in fact all the $L^{p}$ norms with $1 \leq p \leq \infty)$ of the temperature $\theta$ (vorticity for the potential velocity [21]) for SQG (resp., the scalar vorticity $\omega=\nabla^{\perp} \cdot u$ for $2 \mathrm{D}$ Euler). With respect to these socalled Casimirs, the respective Hamiltonians of these systems lie at a negative level of regularity: $\|\theta\|_{\dot{H}^{-1 / 2}}$ for SQG (resp., $\|\omega\|_{\dot{H}^{-1}}=\|u\|_{L^{2}}$ for 2D Euler). As such, much of the discussion presented in this paper concerning the MHD system has an analogue in the context of the SQG [21, 94, 96] and 2D Euler equations [29, 30]. 
2.3.2. Onsager-type dichotomies. Similarly to Onsager's conjecture for the 3D Euler equations, it is natural to analyze the regularity threshold at which weak solutions of (2.19) (in the sense of Definition 2.8) respect the ideal MHD conservation laws for the energy $\mathcal{E}$, the cross helicity $\mathcal{H}_{\omega, B}$, and the magnetic helicity $\mathcal{H}_{B, B}$ [1, 2 . Given a Banach scale used to measure the regularity of weak solutions, we wish to identify a critical/threshold exponent, above which all weak solutions obey the given conservation law (rigidity), while below this exponent there exist weak solutions which violate it (flexibility) 8

Conjecture 2.10 (Onsager-type conjecture for the Elsässer energies).

(a) Any weak solution $(v, B)$ of the ideal MHD system (2.19) belonging $C_{x, t}^{\alpha}$ (resp., $L_{t}^{3} B_{3, \infty, x}^{\alpha}$ ) for $\alpha>1 / 3$, conserves the total energy $\mathcal{E}$ and the cross helicity $\mathcal{H}_{\omega, B}$.

(b) For any $\alpha<1 / 3$ there exist weak solutions $(v, B) \in C_{x, t}^{\alpha}\left(\right.$ resp., $\left.L_{t}^{3} B_{3, \infty, x}^{\alpha}\right)$, which dissipate the total energy $\mathcal{E}$, and for which the cross helicity $\mathcal{H}_{\omega, B}$ is not a constant function of time.

The statement of the corresponding dichotomy for the magnetic helicity is slightly modified, to avoid spaces of negative regularity, which are not consistent with Definition 2.8. In particular, in the flexibility statement, we relax the integrability exponent not the regularity assumptions of the weak solutions.

Conjecture 2.11 (Onsager-type conjecture for the magnetic helicity).

(a) Any weak solution $(v, B)$ of the ideal MHD system (2.19) belonging to $L_{t}^{3} L_{x}^{3}$ (resp., $L_{t}^{3} B_{3, c_{0}, x}^{0}$ ) conserves the magnetic helicity $\mathcal{H}_{B, B}$.

(b) For any $p \in[2,3)$, there exist weak solutions $(v, B) \in L_{t}^{3} L_{x}^{p}$ (resp., $\left.L_{t}^{3} B_{p, \infty, x}^{0}\right)$ for which the magnetic helicity $\mathcal{H}_{B, B}$ is not a constant function of time.

The rigidity parts of the above conjectures are discussed in Section 2.3 .3 below, while progress towards the flexibility parts is outlined in Section [5. As opposed to Onsager's conjecture for 3D Euler, which is by now a theorem, the flexibility statements part (b) of Conjecture 2.10 and part (b) of Conjecture 2.11 remain to date open. We only mention at this stage that for $B \equiv 0$ and any $v$ as constructed in part (b) of Theorem 2.4 the resulting pair $(v, B)$ is a weak solution with the regularity required by the flexible part of Conjecture 2.10, and for which the total energy is dissipated. However, for any such solution the cross helicity and the magnetic helicity are trivial since $B$ is trivial (i.e., identically equal to zero) and thus $\mathcal{H}_{B, B}=\mathcal{H}_{\omega, B}=0$ are constants in time.

2.3.3. The proof of rigidity in Onsager-type conjectures for $M H D$. We start by discussing part (国) of Conjecture 2.10. Recall that the conservation of $\mathcal{E}$ and $\mathcal{H}_{\omega, B}$ is equivalent to the conservation of the Elsässer energies, i.e., the validity of (2.24). Inspecting the momentum equation in (2.23), we see that the only difference to $3 \mathrm{D}$ Euler is that $z_{\mp}$ appears as the transport velocity for the $z_{ \pm}$evolution. With this modification in mind, one may define two fluxes $\Pi_{\kappa+}$ (for the $z_{+}$evolution) and $\Pi_{\kappa-}$ (for the $z_{-}$evolution) in analogy to (2.8). The energy equality again boils down to whether $\lim _{\kappa \rightarrow \infty} \int_{0}^{T} \Pi_{\kappa \pm}=0$ or not. The former question has been

\footnotetext{
${ }^{8}$ See 104 , where this question is posed for general nonlinear, supercritical, Hamiltonian evolution equations.
} 
resolved by repeating the argument in [45, which gives a bound for the commutator $C_{\kappa}\left(z_{\mp}, z_{ \pm}\right)$as in (2.10). This proof was carried through in [26], where it is shown that $\left|\Pi_{\kappa \pm}\right| \lesssim \kappa^{1-3 \alpha}\left\|z_{+}\right\|_{B_{3, \infty}^{\alpha}, x}^{3}+\kappa^{1-3 \alpha}\left\|z_{-}\right\|_{B_{3, \infty}^{\alpha}, x}^{3}$ and thus the conservation of energy and cross helicity holds for any weak solutions $(v, B) \in L_{t}^{3} B_{3, \infty, x}^{\alpha}$ with $\alpha>1 / 3$. We also refer the reader to [100] where the methods of [29] and used to establish the endpoint case for rigidity in Conjecture 2.10 namely $v \in L_{t}^{3} B_{3, c_{0}, x}^{1 / 3}$.

Concerning the rigidity part (国) of Conjecture 2.11, we note that in the integrand of (2.29) is zeroth order in $B$ and $v$. This suggests that justifying (2.29) for weak solutions of (2.19) should require less regularity on $v$ and $B$ than was required in order to justify (2.24). Indeed, it is shown in [26] that $\mathcal{H}_{B, B}$ is conserved by weak solutions of (2.19) as soon as $(v, B) \in B_{3, \infty}^{\alpha}$ with $\alpha>0$, so that the threshold regularity is $\alpha=0$. The idea is as follows. We want to use a version of (2.29), with $B$ replaced by $\mathbb{P}_{\leq \kappa} B$. Then, the conservation in time of $\mathcal{H}_{B, B}$ is equivalent to the vanishing as $\kappa \rightarrow \infty$ (integrated in time) of the magnetic flux term, defined in analogy to (2.8) as

$$
\pi_{\kappa}(v, B):=2 \int_{\mathbb{T}^{3}} \mathbb{P}_{\leq \kappa} B \cdot\left(\mathbb{P}_{\leq \kappa}(v \times B)-\left(\mathbb{P}_{\leq \kappa} v \times \mathbb{P}_{\leq \kappa} B\right)\right) .
$$

Using the commutator estimate of [41] (see (2.10), , one then immediately obtains

$$
\left|\pi_{\kappa}(v, B)\right| \lesssim \kappa^{-3 \alpha}\|v\|_{B_{3, \infty, x}^{\alpha}}\|B\|_{B_{3, \infty, x}^{\alpha}}^{2},
$$

which concludes the proof when $\alpha>0$, upon integrating in time and passing $\kappa \rightarrow \infty$. The endpoint case $\alpha=0$ was reached in [1,76, 100, where it is proven that magnetic helicity is conserved for any weak solution (in the sense of Definition 2.8) as soon as $(v, B) \in L_{x, t}^{3} \cap C_{\text {weak }, t}^{0} L_{x}^{2}$. The slightly sharper statement concerning rigidity for the endpoint Besov space stated in part (国) of Conjecture 2.11 may be achieved by repeating the argument of $[29$ to establish a bound for the magnetic flux at dyadic scales: $\left|\pi_{2^{j}}(v, B)\right| \lesssim \sum_{i=1}^{\infty} 2^{-2 / 3|j-i|}\left\|\mathbb{P}_{\approx 2^{i}} v\right\|_{L^{3}}\left\|\mathbb{P}_{\approx 2^{i}} B\right\|_{L^{3}}^{2}$, which is nearly the same as (2.12), except that the $2^{i}$ term on the right side is absent.

We note that for the 2D MHD equations much stronger types of rigidity may be established (when compared to the 3D case discussed in this paper), and we refer interested readers to 76,78 .

2.3.4. Taylor's conjecture for weak ideal limits. While turbulent low-density plasma configurations are observed to dissipate the total energy $\mathcal{E}$ [50,127, it is commonly accepted knowledge in the plasma physics literature, and an experimental fact, that the magnetic helicity $\mathcal{H}_{B, B}$ is conserved [139] in the infinite conductivity limit. This striking phenomenon manifests itself mathematically as Taylor's conjecture [11,132, 168, 169. Here we discuss its rigorous foundations, following [26, 77.

We start from the viscous and resistive MHD system (2.20), where $\nu, \mu>0$. In analogy to the energy inequality (2.5) for the Navier-Stokes equations, sufficiently smooth solutions of (2.20) satisfy the following energy inequality for the total energy $\mathcal{E}$ defined in (2.21):

$$
\mathcal{E}(t)+\nu \int_{s}^{t}\|\nabla v\|_{L^{2}}^{2}+\mu \int_{s}^{t}\|\nabla B\|_{L^{2}}^{2} d s \leq \mathcal{E}(s)
$$

for $t \geq s$. Based on (2.30) it is classical 69] to build a theory of Leray-Hopf weak solutions for (2.20); these are solutions with $v, B \in C_{\text {weak }, t}^{0} L_{x}^{2} \cap L_{t}^{2} \dot{H}_{x}^{1}$ which obey (2.30) for a.e. $s \geq 0$ and all $t>s$. In physically realistic regimes, we are interested 
in $\nu, \mu \ll 1$; however, in the ideal limit $(\nu, \mu) \rightarrow(0,0)$, 2.30) only gives bounds for the $L_{t}^{\infty} L_{x}^{2}$ norms of $v$ and $B$. Following [77, Definition 1.1] we recall the definition:

Definition 2.12 (Weak ideal limit). Let $\left(\nu_{j}, \mu_{j}\right) \rightarrow(0,0)$ be a sequence of vanishing viscosities and resistivities. Associated to a sequence of divergence free initial data converging weakly $\left(v_{0, j}, B_{0, j}\right) \rightarrow\left(v_{0}, B_{0}\right)$ in $L^{2}\left(\mathbb{T}^{3}\right)$, let $\left(v_{j}, B_{j}\right)$ be a sequence of Leray-Hopf weak solutions of (2.20). Any pair of functions $(v, B)$ such that $\left(v_{j}, B_{j}\right) \stackrel{*}{\rightarrow}(v, B)$ in $L^{\infty}\left(0, T ; L^{2}\left(\mathbb{T}^{3}\right)\right)$, are called a weak ideal limit of the sequence $\left(v_{j}, B_{j}\right) 9$

Taylor's conjecture states that weak ideal limits of MHD Leray-Hopf weak solutions conserve magnetic helicity. This was proven recently in [77, and we recall the statement:

Theorem 2.13 (Taylor's conjecture; Theorem 1.2 in [77]). Suppose $(u, B) \in L_{t}^{\infty} L_{x}^{2}$ is a weak ideal limit of a sequence of Leray-Hopf weak solutions. Then $\mathcal{H}_{B, B}$ is a constant function of time. In particular, finite energy weak solutions of the ideal MHD equations (2.19), which are weak ideal limits, conserve magnetic helicity.

At the heart of the proof of Theorem 2.13 given in [77] (where they also consider domains that are not simply connected) lies the observation that for $\mu, \nu>0$, by interpolation we have that MHD Leray-Hopf weak solutions lie in $L_{t, x}^{10 / 3}$, which is a stronger space than the $L_{t, x}^{3}$ which is required in part (国) of Conjecture 2.11. Thus, in analogy to the proof of (2.3) discussed earlier, at fixed $\mu_{j}, \nu_{j}>0$ one may justify the magnetic helicity equality

$$
\mathcal{H}_{B_{j}, B_{j}}(t)+2 \mu_{j} \int_{0}^{t} \int_{\mathbb{T}^{3}}\left(\nabla \times B_{j}\right) \cdot B_{j}=\mathcal{H}_{B_{j}, B_{j}}(0) .
$$

The bounds provided by (2.30) immediately imply that the second term on the left side of (2.31) may be bounded by $\left(t \mu_{j}\right)^{1 / 2} \mathcal{E}(0)$, and thus this term vanishes in the ideal limit $\mu_{j} \rightarrow 0$. The proof of Theorem 2.13 is then concluded by showing that due to the compactness of the embedding $L^{2} \subset \dot{H}^{-1 / 2}$ for the subsequential limits in Definition 2.12 we have $B_{j} \rightarrow B$ strongly in $C_{t} \dot{H}_{x}^{-1 / 2}$, and so $A_{j} \rightarrow A$ strongly in $C_{t} \dot{H}_{x}^{1 / 2}$. By the definition of magnetic helicity in (2.27), this information is sufficient to show that $\mathcal{H}_{B_{j}, B_{j}} \rightarrow \mathcal{H}_{B, B}$ uniformly in time, and thus letting $j \rightarrow \infty$ in (2.31) concludes the proof 10

Remark 2.14. In closing, we note that Theorem 2.13 does not contradict part (b) of Conjecture 2.11, Theorem 2.13 does indeed show that if $(v, B)$ is a weak ideal limit (cf. Definiton 2.12) and also a weak solution of the ideal MHD equations (cf. Definiton 2.8), then it conserves magnetic helicity, although it may have regularity as weak as $L_{t}^{\infty} L_{x}^{2}$. This is far less spatial integrability than the $L_{t}^{3} L_{x}^{3}$ condition required in part (a) of Conjecture 2.11] which points to the fact that weak ideal limits are

\footnotetext{
${ }^{9}$ Note however that a weak ideal limit $(v, B)$ need not be a weak solution of the ideal MHD equations in the sense of Definition 2.8, for the same reason that a vanishing viscosity limit of Leray-Hopf weak solutions of the 3D Navier-Stokes equations need not be a weak/distributional solution of the 3D Euler equations; only measure valued solutions are known to be achieved as subsequential limits 66.

${ }^{10}$ We note that similar arguments were previously used to prove the conservation of the Hamiltonian for the 2D Euler 30] and 2D SQG equations [43, for weak solutions which arise from vanishing viscosity limits.
} 
seeing a ghost of the energy inequality (2.30). Nonetheless, the flexible part of Conjecture 2.11 is that there may exist weak solutions of ideal MHD which do not arise in the vanishing viscosity/resistivity limit, leaving open the possibility that for such solutions $\mathcal{H}_{B, B}$ is not constant in time. This specific result is established in Theorem 5.2 below.

\section{Convex integration And Nash schemes for the Euler equations}

While the rigid part of Onsager's conjecture (part (国) of Theorem 2.4) has been essentially understood since the 1990s, systematic progress towards the resolution of the flexible part (b) did not occur until the 2010s and the groundbreaking works [55, 58 of De Lellis and Székelyhidi Jr. These works have developed the mathematical framework of the $L_{t, x}^{\infty}$ and the $C_{t, x}^{0}$ flavors of convex integration in fluid dynamics, and have laid out some of the key ideas which have eventually led to the solution of the flexible part of Onsager's conjecture by Isett 93. (in the context of solutions with compact support in time); and in a subsequent work by Buckmaster, De Lellis, Székelyhidi, and Vicol [20] (for dissipative weak solutions). This sequence of developments has already been discussed in great detail in the review papers $[22,57$, 60, 61, 162, so in this section, we only give a succinct presentation. We emphasize that the $L_{t, x}^{\infty}$ convex integration scheme used to prove Theorems 3.1 and 3.2 has a number of key similarities to, but is also conceptually different from, the $C_{t, x}^{\alpha}$ Nash-type convex integration method used to prove Theorems 3.3, 3.4, and 3.5.

3.1. The flexible part of Onsager's conjecture: First paradoxical examples. In the seminal work [147, Scheffer demonstrated the existence of nontrivial weak solutions of the $2 \mathrm{D}$ Euler system (2.16), which lie in $L_{x, t}^{2}$ and have compact support in time and space! Strictly speaking the weak solutions of Scheffer are not dissipative, as dissipative solutions are required to have nonincreasing energy; nonetheless, [147] is considered to be the first result concerning the flexible part (b)), of Onsager's conjecture.

A different construction of a nontrivial weak solution to the 2D Euler equations, which are periodic in space and have compact support in time, was given by Shnirelman in [153. The existence of dissipative weak solutions to the Euler equations was first proven by Shnirelman in [152, where he constructs weak solutions which lie in $L_{t}^{\infty} L_{x}^{2}$.

These results, which were initially referred to as the Scheffer-Shnirelman paradox, represent not just a proof of nonuniqueness for weak solutions to the Euler equations, but a drastic failure of determinism within this class of solutions.

3.2. The $L_{x, t}^{\infty}$ results. The first example of a bounded in space and time, dissipative weak solution of the Euler equations (one for which the kinetic energy is a nonincreasing function of time), in any dimension $n \geq 2$, was obtained in a groundbreaking work by De Lellis and Székelyhidi Jr. [55]. Their main result is:

Theorem 3.1 (Theorem 4.1 in [55]). For any open bounded space-time domain $\Omega \subset \mathbb{R}^{n} \times \mathbb{R}$, there exists a weak solution of the Euler equations (2.16) $(v, p) \in$ $L^{\infty}\left(\mathbb{R}^{n} \times \mathbb{R}\right)$, in the sense of Definition 2.3 , such that $|v(x, t)|=1$ for a.e. $(x, t) \in \Omega$, and $v(x, t)=p(x, t)=0$ for a.e. $(x, t) \in \Omega^{c}$. Moreover, there exists a sequence of functions $\left(v_{q}, p_{q}, f_{q}\right) \in C_{0}^{\infty}(\Omega)$ such that:

- $\partial_{t} v_{q}+\operatorname{div}\left(v_{q} \otimes v_{q}\right)+\nabla p_{q}=f_{q}$, and $\nabla \cdot v_{q}=0$,

- $f_{q} \rightarrow 0$ in $H^{-1}$ as $q \rightarrow \infty$, 
- $\left\|v_{q}\right\|_{L^{\infty}}+\left\|p_{q}\right\|_{L^{\infty}}$ is uniformly bounded in $q$,

- $\left(v_{q}, p_{q}\right) \rightarrow(v, p)$ in $L^{r}$ for any $r<\infty$.

The first part of the theorem establishes the existence of a weak solution which is compactly supported in space and time, while the second part is a manifestation of the proof: the limiting weak solution $(v, p)$ is obtained from a smooth approximating sequence $\left(v_{q}, p_{q}\right)$, which solves a relaxed Euler system, whose right side $f_{q}$ vanishes in a weak sense as $q \rightarrow \infty$. This paper also introduced the ideas of a subsolution and of a Reynolds stress for the Euler system (2.16). Maybe more important than the result itself, which was later improved by the same authors, is the fact that 55 ] relates the construction of paradoxical weak solutions of the Euler equations with a classical technique in geometry, convex integration, and the notion of $h$-principles for soft partial differential equations.

The method of convex integration can be traced back to the work of Nash, who used it to construct exotic counterexamples to the $C^{1}$ isometric embedding problem [135. The method was later refined by Gromov [86], and it evolved into a general method for solving soft/flexible geometric partial differential equations [70]. In the influential paper [134, Müller and Šverák adapted convex integration to the theory of differential inclusions (see also [102]), leading to renewed interest in the method as a result of its greatly expanded applicability. Inspired by the works [102, 134, and building on the plane-wave analysis introduced by Tartar [165, 166] and Di Perna [64, De Lellis and Székelyhidi Jr., in [55], applied convex integration in the context of bounded weak solutions to the Euler equations.

We refer the interested reader to the review papers [57,61, 162] for a detailed discussion connecting convex integration in the context of differential inclusions, and also $h$-principles, to the type of constructions that were initiated by [55]. In particular, analyzing the toy example presented in [57, Section 5.1] is particularly accessible, yet insightful.

The work [55], has since been extended and adapted by various authors to various problems arising in mathematical physics, including [6, 27, 36, 39, 46, 47, 56, 155, 161, 163, 174, just to mention a few. Here we single out the work [56] which considers the question of whether imposing additional admissibility criteria on the weak solutions of the Euler equations could rule out the construction of examples such as those in Theorem 3.1. Physically motivated admissibility criteria, based on energetic arguments such as those discussed in Section 2 (ordered by least restrictive to most restrictive) include the following.

(i) Weak energy inequality: the kinetic energy satisfies $\mathcal{E}(t) \leq \mathcal{E}(0)$ for all $t>0$.

(ii) Strong energy inequality: the kinetic energy satisfies $\mathcal{E}(t) \leq \mathcal{E}(s)$ for all $t>s>0$.

(iii) Local energy inequality: the distribution $\partial_{t} \frac{|v|^{2}}{2}+\operatorname{div}\left(v\left(p+\frac{|v|^{2}}{2}\right)\right)$, for $v \in$ $L_{\mathrm{loc}, t, x}^{3}$, is nonnegative.

The main result of [56] may be summarized as follows.

Theorem 3.2 (Theorem 1 in [56]). For any dimension $n \geq 2$, there exists bounded, compactly supported divergence-free initial data $v_{0} \in L_{x}^{\infty} \cap L_{x}^{2}$, for which there exist infinitely many weak solutions $v \in L_{x, t}^{\infty} \cap C_{t}^{0} L_{x}^{2}$ of the Euler equations, such that the admissibility conditions (ii), (iii), and (iii) hold. 
This result shows that "wild" weak solutions of the Euler equations (as constructed by Theorem 3.1 and Theorem 3.2) cannot be ruled by the local energy inequality. Indeed, the Euler equations are not scalar conservation laws!

3.3. The $C_{x, t}^{0+}$ result: A Nash-type convex integration scheme. The $L_{x, t}^{\infty}$ constructions described in Section 3.2 are based on writing the Euler equations as a differential inclusion, and then applying a machinery from Lipschitz differential inclusions, which either uses a Baire-category argument or, equivalently, an explicit convex integration approach. These methods face a serious difficulty in constructing continuous weak solutions of (2.16), since it seems impossible to extract a uniform continuity estimate for approximating sequences $\left(v_{q}, p_{q}\right)$. The breakthrough was made by De Lellis and Székelyhidi Jr. in their seminal papers [58, 59, where they developed a new convex integration scheme, motivated by and resembling in part the earlier schemes of Nash and Kuiper [108, 135]. In [58, De Lellis and Székelyhidi Jr. prove the existence of continuous weak solutions $v$ to the Euler equations satisfying a prescribed kinetic energy profile, which in particular may be decreasing:

Theorem 3.3 (Theorem 1.1 in [58). Assume $e:[0,1] \rightarrow(0, \infty)$ is a smooth function. Then there is a continuous vector field $v: \mathbb{T}^{3} \times[0,1] \rightarrow \mathbb{R}^{3}$ and a continuous scalar field $p: \mathbb{T}^{3} \times[0,1] \rightarrow \mathbb{R}$ which solve the incompressible Euler equations (2.16) in the sense of distributions, and such that

$$
e(t)=\frac{1}{2} \int_{\mathbb{T}^{3}}|v(\cdot, t)|^{2}
$$

for all $t \in[0,1]$.

The proof of Theorem 3.3 departs from the arguments based on functional analysis, which were used to construct bounded weak solutions, and implements a hard analysis scheme, in which the constructions of the building blocks are not plane waves anymore, instead they are adapted to the geometry of steady states of the Euler equations (Beltrami flows), and the estimates involve precise singular integral bounds and Schauder estimates.

3.3.1. Overview of the proof of Theorem 3.3 and of Nash-type convex integration schemes. The summary given here is similar to the one we have given in $[22$, Section 4.1].

For each $q \in \mathbb{N}_{0}$ by induction one constructs smooth functions $\left(v_{q}, \stackrel{\circ}{R}_{q}\right)$, which solve the Euler-Reynolds system

$$
\begin{aligned}
\partial_{t} v_{q}+\operatorname{div}\left(v_{q} \otimes v_{q}\right)+\nabla p_{q} & =\operatorname{div} \stackrel{\circ}{R}_{q}, \\
\operatorname{div} v_{q} & =0 .
\end{aligned}
$$

The pressure $p_{q}$ is always given by $p_{q}=(-\Delta)^{-1} \operatorname{div} \operatorname{div}\left(v_{q} \otimes v_{q}-\stackrel{\circ}{R}_{q}\right)$. The stress $\stackrel{\circ}{R}_{q}$ is symmetric and has zero trace (since its trace is absorbed in to the pressure term). The goal is to construct the sequence $\left(v_{q}, \stackrel{\circ}{R}_{q}\right)$ such that $\stackrel{\circ}{R}_{q}$ converges uniformly to 0 as $q \rightarrow \infty$, and that at the same time the sequence $v_{q}$ converges uniformly to a Hölder continuous weak solution to the Euler equations, which satisfies (3.1). The iterates $\left(v_{q}, \stackrel{\circ}{R}_{q}\right)$ constructed via the convex integration scheme are approximately the spatial averages of the final solution $v$ at length scales $\lambda_{q}^{-1}$, which are decreasing with $q$. In view of the analogy to theories in fluid turbulence, one refers to the symmetric tensor $\stackrel{\circ}{R}_{q}$ as the Reynolds stress. 
At each inductive step, the goal is to design a perturbation

$$
w_{q+1}=v_{q+1}-v_{q}
$$

such that the new velocity $v_{q+1}$ and pressure $p_{q+1}$ solve the Euler-Reynolds system (3.2) at level $q+1$, but with a smaller Reynolds stress $\stackrel{\circ}{R}_{q+1}$. Subtracting the equations for $v_{q+1}$ and $v_{q}$, we obtain the following decomposition of the Reynolds stress at level $q+1$ :

$$
\begin{aligned}
\operatorname{div} \stackrel{\circ}{R}_{q+1}-\nabla\left(p_{q+1}-p_{q}\right) & \\
= & \underbrace{\operatorname{div}\left(w_{q+1} \otimes w_{q+1}+\stackrel{\circ}{R}_{q}\right)}_{\text {oscillation error }}+\underbrace{\partial_{t} w_{q+1}+v_{q} \cdot \nabla w_{q+1}}_{\text {transport error }}+\underbrace{w_{q+1} \cdot \nabla v_{q}}_{\text {Nash error }} .
\end{aligned}
$$

Note that not all the terms on the right side of (3.4) are written in divergence form, which necessitates the use of a negative one order linear Fourier multiplier operator $\mathcal{R}$ which formally acts as $\operatorname{div}^{-1}$ and outputs symmetric traceless matrices 11

For an increasing sequence of frequency parameters $\left\{\lambda_{q}\right\}_{q \geq 0}$, the approximate solutions at level $\left(v_{q}, \stackrel{\circ}{R}_{q}\right)$ are essentially localized at Fourier frequencies $\lesssim \lambda_{q}$. On the other hand, the perturbation $w_{q+1}=v_{q+1}-v_{q}$ is constructed as a sum of highly oscillatory building blocks (denoted by $W_{\xi}$ in (3.5) below) which live at the high frequency $\lambda_{q+1} \gg \lambda_{q}$.

Roughly speaking, the principal part of the perturbation, which we label as $w_{q+1}^{(p)}$, will be of the form

$$
w_{q+1}^{(p)} \approx \sum_{\xi} a_{\xi}\left(\stackrel{\circ}{R}_{q}\right) W_{\xi},
$$

where $\xi$ ranges over a finite set, $W_{\xi}$ represents the building blocks oscillating at frequency $\lambda_{q+1}$, and the coefficient functions $a_{\xi}$ are chosen such that

$$
\sum_{\xi} a_{\xi}^{2}\left(\stackrel{\circ}{R}_{q}\right) f_{\mathbb{T}^{3}} W_{\xi} \stackrel{\otimes}{\otimes} W_{\xi}=-\stackrel{\circ}{R}_{q} .
$$

Here $\otimes$ denotes the trace-free part of the tensor product. The amplitude functions $a_{\xi}$ are designed in order to obtain a cancellation between the low frequencies of the quadratic term $w_{q+1}^{(p)} \otimes w_{q+1}^{(p)}$ and the old Reynolds stress error $\stackrel{\circ}{R}_{q}$, thereby reducing the size of the low frequency part of the oscillation error. More precisely, if we take into account that $W_{\xi} \otimes W_{\xi^{\prime}}$ are chosen to have no low frequency (meaning $\lambda_{q}$ ) contribution when $\xi \neq \xi^{\prime}$, the need to minimize the low frequency part of the oscillation error, $\stackrel{\circ}{R}_{q}+\mathbb{P}_{\lesssim \lambda_{q}}\left(w_{q+1}^{(p)} \otimes w_{q+1}^{(p)}\right)$, dictates the choice (3.6).

To leading order, with respect to the large parameter $\lambda_{q+1}$, the zero mean $\mathbb{T}^{3}$ periodic building blocks $W_{\xi}$ are chosen to be solutions of the stationary Euler equations, i.e., they satisfy $\operatorname{div}\left(W_{\xi} \otimes W_{\xi}\right)+\nabla P=0$ for a suitable pressure $P$, and $\operatorname{div} W_{\xi}=0$. The importance of this choice becomes apparent when one takes into account the ansatz (3.5), and uses it to compute the high frequency part of the oscillation error on the right side of (3.4), namely $\mathbb{P}_{\gg \lambda_{q}}\left(w_{q+1}^{(p)} \otimes w_{q+1}^{(p)}\right)$. The building blocks used in [58,59], are the so-called Beltrami waves, which are families

${ }^{11}$ For $v$ of zero mean one may define

$$
(\mathcal{R} v)^{k \ell}=\Delta^{-1}\left(\partial_{k} v^{\ell}+\partial_{\ell} v^{k}\right)-\frac{1}{2}\left(\delta_{k \ell}+\partial_{k} \partial_{\ell} \Delta^{-1}\right) \operatorname{div} \Delta^{-1} v .
$$


of complex eigenfunctions of the curk operator at the same eigenvalue, $\lambda_{q+1}$. Starting with [51, the later works [20,92,93] use the so-called Mikado flows, which are straight pipe flows with pairwise disjoint supports (see Section 3.5). These building blocks are used in an analogous fashion to the Nash twists and Kuiper corrugations employed in the $C^{1}$ embedding problem 108, 135.

The principal part of the perturbation presented in 3.5 needs to be modified in order to minimize the transport error in (3.4), i.e., to ensure it is the divergence of a small Reynolds stress. This is achieved by flowing the building blocks $W_{\xi}$ along the ODE flow generated by $v_{q}$ (we will return to this issue in Section 3.4). Additionally, in order to ensure that $w_{q+1}$ is divergence free, one introduces a corrector $w_{q+1}^{(c)}$ which ensures that $w_{q+1}=w_{q+1}^{(p)}+w_{q+1}^{(c)}$ is divergence free. The size of this incompressibility corrector $w_{q+1}^{(c)}$ is much smaller than the size of $w_{q+1}^{(p)}$, roughly by a factor of $\lambda_{q} \lambda_{q+1}^{-1}$, because the building blocks $W_{\xi}$ are divergence-free by definition, and the $a_{\xi}$ oscillate at the old frequency, $\lambda_{q}$.

In order to ensure that the inductive scheme converges to a Hölder continuous velocity $v$ with Hölder exponent $>0$, the amplitude of the perturbation is required to satisfy the bound

$$
\left\|w_{q+1}\right\|_{C_{t, x}^{0}} \leq \lambda_{q+1}^{-\beta}
$$

for some $\beta>0$. Here, we note that it is convenient to use a superexponentially growing sequence of frequencies $\lambda_{q}$ which obeys $\lambda_{q+1} \approx \lambda_{q}^{b}$, for some $b>1$. In view of (3.6), this necessitates that the Reynolds stress $\stackrel{\circ}{R}_{q}$ obeys the estimate

$$
\left\|\stackrel{\circ}{R}_{q}\right\|_{C_{t, x}^{0}} \leq \lambda_{q+1}^{-2 \beta} \text {. }
$$

Consistent with the definition

$$
v_{q}=v_{0}+\sum_{q^{\prime}<q} w_{q^{\prime}},
$$

and with the bound (3.7), the scheme of [58] also propagates the estimate

$$
\left\|\nabla v_{q}\right\|_{C_{t, x}^{0}} \lesssim \lambda_{q}^{1-\beta} \text {. }
$$

It is not hard to see that if the bounds (3.7)-(3.10) are propagated throughout the scheme, then as $q \rightarrow \infty$ we have that $\left(v_{q}, \stackrel{\circ}{R}_{q}\right) \rightarrow(v, 0)$ uniformly, where $v$ is a Hölder continuous weak solution of the Euler equations. Indeed, for any $\theta \in(0, \beta)$, the following series of increments is summable:

$$
\begin{aligned}
\sum_{q \geq 0}\left\|w_{q+1}\right\|_{C_{t}^{0} C_{x}^{\theta}} & \lesssim \sum_{q \geq 0}\left\|w_{q+1}\right\|_{C_{t, x}^{0}}^{1-\theta}\left\|\nabla w_{q+1}\right\|_{C_{t, x}^{0}}^{\theta} \\
& \lesssim \sum_{q \geq 0} \lambda_{q+1}^{-\beta(1-\theta)} \lambda_{q+1}^{\theta(1-\beta)} \lesssim \sum_{q \geq 0} \lambda_{q+1}^{\theta-\beta} \lesssim 1
\end{aligned}
$$

where the implicit constant is universal. Thus, we may define a limiting function $v=\lim _{q \rightarrow \infty} v_{q}$ which lies in $C^{0}\left([0,1] ; C^{\theta}\right)$. Moreover, $v$ is a weak solution of the Euler equation (2.16), since by (3.8) we have that $\lim _{q \rightarrow \infty} \stackrel{\circ}{R}_{q}=0$ in $C_{t, x}^{0}$, and $v_{q} \otimes v_{q} \rightarrow v \otimes v$ strongly in $C_{t, x}^{0}$.

The main work is now to prove that for a velocity perturbation $w_{q+1}$ of the form (3.5), and with amplitude functions that satisfy (3.6), the bounds stated in (3.7)(3.10) are indeed attainable inductively for all $q \geq 1$. We note that if the building 
blocks are normalized so that $\left\|W_{\xi}\right\|_{C_{t, x}^{0}} \approx 1$, then it follows from (3.5)-(3.6) and (3.8) that the principal part of the velocity increment already satisfies the bound (3.7). Since the incompressibility corrector is even smaller, (3.7) is expected to hold. The difficult part is to prove (3.8). In view of (3.4), this amounts to bounding the oscillation error, the transport error, and the Nash error. This is the hard analysis part of the construction.

As a demonstration of the typical scalings present in convex integration schemes for the Euler equations, let us consider the Nash error. Heuristically, since $w_{q+1}$ is of frequency $\lambda_{q+1} \gg \lambda_{q^{\prime}}$ for every $q^{\prime} \leq q$, and by appealing to (3.9), we have that $w_{q+1} \cdot \nabla v_{q}$ lives at frequency $\lambda_{q+1}$, and thus

$$
\left\|\mathcal{R}\left(w_{q+1} \cdot \nabla v_{q}\right)\right\|_{C_{t, x}^{0}} \lesssim \lambda_{q+1}^{-1}\left\|w_{q+1}\right\|_{C_{t, x}^{0}}\left\|\nabla v_{q}\right\|_{C_{t, x}^{0}},
$$

where we recall that $\mathcal{R}$ is a -1 order Fourier multiplier which inverts the divergence operator. Applying (3.7) and (3.10), for $\beta \in(0,1)$ we obtain

$$
\left\|\mathcal{R}\left(w_{q+1} \cdot \nabla v_{q}\right)\right\|_{C_{t, x}^{0}} \lesssim \lambda_{q+1}^{-1-\beta} \lambda_{q}^{1-\beta} \approx \lambda_{q+2}^{-2 \beta} \lambda_{q}^{1-\beta-b(1+\beta)+2 \beta b^{2}}
$$

by using that $\lambda_{q+1} \approx \lambda_{q}^{b}$. Thus, in order to ensure that $\stackrel{\circ}{R}_{q+1}$ satisfies the bound (3.8) with $q$ replaced by $q+1$, we require that for $\beta \in(0,1)$ and $b>1$ we have

$$
1-\beta-b(1+\beta)+2 \beta b^{2}=(1-b)(1-\beta-2 \beta b)<0 .
$$

Thus, from this simple heuristic, we see that if $b>1$ is taken to be arbitrarily close to 1 , then the Hölder regularity exponent $\beta$ may be taken to be arbitrarily close to the Onsager-critical Hölder regularity exponent, i.e., $\beta<1 / 3$.

The construction described above provides a clear enemy towards reaching the desired Onsager $1 / 3$ threshold: the transport and oscillation errors in (3.4). Designing a $w_{q+1}$ which minimizes these two errors simultaneously turns out to be a very difficult problem. This realization stimulated a series of advancements, through the works [15, 17, 18, 51, 90, in which the authors incorporated more and more of the specifics of the 3D Euler equation into the convex integration scheme (by designing better $W_{\xi}$ and $a_{\xi}$ ), in order to obtain higher and higher Hölder regularity exponents. We mention a couple of these developments next.

3.4. Climbing the Onsager ladder. The first breakthrough after [58,59] was to produce a dissipative weak solution of the Euler system with a Hölder regularity exponent $\beta$ with $\beta<1 / 5$. This was achieved by Isett 90 and later simplified in [19] by Buckmater, De Lellis, and Székelyhidi, the two papers resulting in the joint work [17. The main improvement comes from obtaining a better bound for the transport error in (3.4). In the proof of Theorem 3.3 one did not keep track of precise estimates for the material derivative of the Reynolds stress $\left(\partial_{t}+v_{\ell} \cdot \nabla\right) \stackrel{\circ}{R}_{q}$. Here $v_{\ell}$ is a mollification of $v_{q}$ at a length scale $\ell$ which lies in between $\lambda_{q+1}^{-1}$ and $\lambda_{q}^{-1} 12$ The realization of the $1 / 5$ schemes is that material derivatives are better behaved than either regular spatial or temporal derivatives: due to classical ODE

\footnotetext{
${ }^{12} \mathrm{An}$ inherent issue associated with convex integration schemes is that in order to control $n$th order derivatives of the perturbation $w_{q+1}$, one needs control derivatives on $v_{q}$ of an order strictly greater than $n$. In order to avoid this loss of derivative, one replaces $v_{q}$ by a mollified velocity field $v_{\ell}$ and the stress $\stackrel{\circ}{R}_{q}$ by a mollified stress $\stackrel{\circ}{R}_{\ell}$, where the mollification parameter $\ell \in\left(\lambda_{q+1}^{-1}, \lambda_{q}^{-1}\right)$ is to be chosen suitably. This argument was already required in the $C_{x, t}^{0+}$ schemes described in Section 3.3
} 
arguments, material derivatives should cost a factor proportional to the Lipschitz norm of $v_{q}$, i.e., $\lambda_{q}^{1-\beta}$ in view of (3.10). Compare this with a spatial derivative, whose cost is $\lambda_{q} \gg \lambda_{q}^{1-\beta}$. Taking advantage of this observation, one can improve the estimate on the material derivative of $\stackrel{\circ}{R}_{q}$, and thus improve the bounds for the transport error. Optimizing this new transport bound with the oscillation error yields the improved $1 / 5$-Hölder exponent.

The $1 / 5$-scheme is very versatile, and it was successfully used to construct weak solutions of 3D Euler with compact support on the whole space [95, to establish the nonuniqueness of weak solutions for the 3D quasi-geostrophic equations [137, the 2D SQG equations [21,94, active scalar equations with nonodd constitutive laws 96, and the hypodissipative Navier-Stokes equations [40] (this result was later improved in 62] to take into account the techniques used to prove Theorem 3.5 below).

In [15, the first author noted that one can construct infinitely many weak solutions of (2.16) whose Hölder regularity exponent with respect to the space variable can be taken to be any $\beta$ with $\beta<1 / 3$, but only almost everywhere in time. This new scheme concentrates the transport and oscillation errors on a zero-measure set of times. By taking advantage of this idea and by using a delicate bookkeeping scheme, Buckmaster, De Lellis, and Székelyhidi [18 constructed nonconservative solutions in the space $L_{t}^{1} C_{x}^{1 / 3-}$.

3.5. Resolution of the flexible side of Onsager's conjecture. The flexible side of Onsager's conjecture was finally resolved by Isett in 93, who proved the existence of nonconservative weak solutions of $3 \mathrm{D}$ Euler in the regularity class $C_{x, t}^{\beta}$, for any $\beta<1 / 3$ :

Theorem 3.4 (Theorem 1 in [93]). For any $\beta \in(0,1 / 3)$ there exists a nonzero weak solution $v \in C^{\beta}\left(\mathbb{T}^{3} \times \mathbb{R}\right)$, such that $v$ vanishes identically outside of a finite interval.

The proof of Isett builds upon the ideas in the above mentioned works, and utilizes two new key ingredients. The first, is the usage of Mikado flows which were introduced earlier by Daneri and Székelyhidi [51. These are a rich family of pressureless stationary solutions of the 3D Euler equation (straight pipe flows), which have a better (when compared to Beltrami flows) self-interaction behavior in the oscillation error, when they are advected by a mean flow. The second key ingredient is that prior to adding the convex integration perturbation $w_{q+1}$, it is very useful to replace the approximate solution $\left(v_{q}, \stackrel{\circ}{R}_{q}\right)$ with another pair $\left(\bar{v}_{q}, \stackrel{\circ}{R}_{q}\right)$, which has the property that $\bar{v}_{q}$ is close to $v_{q}$, but more importantly that $\stackrel{\circ}{R}_{q}(t)$ vanishes on every other interval of size $\approx\left\|\nabla v_{q}\right\|_{C^{0}}^{-1}$ within $[0, T]$. The velocity field $\bar{v}_{q}$ (and consequently also the stress $\stackrel{\circ}{R}_{q}$ ) is obtained by smoothly gluing together exact solutions of the Euler equations, whose initial data are chosen to precisely match $v_{q}$ at suitably spaced instances of time. A proto-version of this gluing scheme may already be found in the work of Shnirelman [153], who however works with Dirac masses in time, which produce unacceptable errors. In turn, working with the glued velocity and stress $\left(\bar{v}_{q}, \stackrel{\circ}{R}_{q}\right)$ results in a major improvement of the size of the oscillation error in the convex integration step since different Mikado flows 
have disjoint supports, and thus do not interact on the time scale dictated by the Lipschitz norm of the mean flow $v_{\ell}$.

The weak solutions constructed by Isett 93, are not strictly dissipative. This issue was resolved in the paper [20] by Buckmaster, De Lellis, Székelyhidi, and Vicol, who prove the precise statement of part (b) of Onsager's conjecture:

Theorem 3.5 (Theorem 1.1 in [20]). Let e $:[0, T] \rightarrow \mathbb{R}$ be a strictly positive smooth function. For any $\beta \in(0,1 / 3)$ there exists a weak solution $v \in C^{\beta}\left(\mathbb{T}^{3} \times[0, T]\right)$ of the Euler equations (2.16), whose kinetic energy at time $t \in[0, T]$ equals $e(t)$.

We note that in 92 , Isett showed that one can further optimize the schemes of [20,93] in order to construct nonconservative weak solutions to the Euler equations that lie in the intersection of all Hölder spaces $C^{\beta}$ for $\beta<1 / 3$.

3.6. Some open problems in the context of 3D Euler. Although the weak solutions constructed in Theorem 3.5 may be constructed to satisfy both the weak (ii) and the strong (iii) energy inequality mentioned earlier in Theorem 3.2, they do not satisfy the local in space and time version of the energy inequality (iii). In this direction, the recent results [91] and [54] achieve the regularity exponents $\beta<1 / 15$, and $\beta<1 / 7$, respectively. Extending these results to the full range $\beta<1 / 3$ remains open (see also [22, Problem 3]).

It is also an open problem to determine whether there exist nonconservative weak solutions to the Euler equations that have Hölder exponent exactly equal to 1/3. Such a result would not be in contradiction with [29] (see also [22, Problem 4]).

At the moment of writing of this article, it is not known how to construct nonunique weak solutions of the 3D Euler equations with $C_{x, t}^{\beta}$ regularity, for some $\beta \in(1 / 3,1)$. It appears that such a result would require fundamental new ideas, beyond the ideas provided by the convex integration schemed described earlier. Lastly, we note that Conjectures 2.6 and 2.7 remain open.

\section{Intermittent COnVex integration for the Navier-Stokes equations}

In the previous section we have described various flexibility results obtained for the $3 \mathrm{D}$ Euler equations via the $L_{t, x}^{\infty}$ convex integration technique and the $C_{t, x}^{\alpha}$ Nashtype convex integration scheme. Both produce infinitely many weak solutions of (2.16) which are bounded in space and time. For this reason, these methods cannot be used to produce finite energy weak solutions of 3D Navier-Stokes equations (cf. Definition 2.2). Indeed, as discussed in Section 2.1.1, if $v$ is a weak solution of (2.1) such that $v \in L_{t, x}^{\infty}$, then it is automatically smooth and thus unique 75]. Thus, in order to extend the applicability of convex integration techniques to the Navier-Stokes system requires a new approach.

Building on the Nash-type convex integration method in [58 and drawing inspiration from the experimental reality that fully developed turbulent flows are intermittent 13 [82,133, a new technique (which we call intermittent convex integration) was developed by the authors in [23. In physical space, intermittency

\footnotetext{
${ }^{13}$ Broadly speaking, intermittency is characterized as a deviation from the Kolmogorov 1941 scaling laws, which were derived under the assumptions of homogeneity and isotropy. Experimentally, it is seen that these assumptions need not hold at large Reynolds numbers. A common signature of intermittency is that the $p$ th order structure function exponents $\zeta_{p}$ deviate from the Kolmogorov predicted value of $p / 3$ for $p \neq 3$ [3 $99,126,136$.
} 
causes concentrations that result in the formation of intermittent peaks. In frequency space, intermittency smears frequencies. Analytically, intermittency has the effect of saturating Bernstein inequalities between different $L^{p}$ spaces [35]. In the context of convex integration, intermittency reduces the strength of the linear dissipative term $\nu \Delta v$ in order to ensure that the nonlinear term $\operatorname{div}(v \otimes v)$ dominates. We refer the reader to [22, Sections 2 and 7], where more heuristics (and references) about intermittency are presented and the fine details of the proof in 23 are given.

The goal of this section is to show how intermittent convex integration may be used to prove the nonuniqueness of weak/mild solutions (cf. Definition 2.2) to the 3D Navier-Stokes and to present a number of variants and improvements of the method from [23] which were recently obtained in [14, 16, 48, 122, 123, 129, 130. The application of intermittent convex integration to the MHD system is given in Section 5 below.

4.1. Nonuniqueness of weak solutions with finite kinetic energy. In [23], we have proven the existence of infinitely many weak/mild solutions $v$ of the 3D NavierStokes equations (2.1), in the sense of Definiton 2.2, with a prescribed kinetic energy profile:

Theorem 4.1 (Theorem 1.2 in [23]). There exists $\beta>0$, such that for any nonnegative smooth function $e(t):[0, T] \rightarrow[0, \infty)$ and any $\nu \in(0,1]$, there exists a weak solution of the Navier-Stokes equations

$$
v \in C^{0}\left([0, T] ; H^{\beta}\left(\mathbb{T}^{3}\right)\right) \cap C^{0}\left([0, T] ; W^{1,1+\beta}\left(\mathbb{T}^{3}\right)\right),
$$

such that $\frac{1}{2} \int_{\mathbb{T}^{3}}|v(x, t)|^{2} d x=e(t)$ holds for all $t \in[0, T]$.

We emphasize that the weak solutions constructed in Theorem4.1 are not LerayHopf weak solutions, whose uniqueness remains famously one of the most challenging questions in fluid mechanics 98 .

Theorem 4.1 shows in particular that $v \equiv 0$ is not the only weak/mild solution which vanishes at a time slice, thereby implying the nonuniqueness of solutions in the sense of Definiton 2.2 (note that within the class of Leray-Hopf weak solutions 0 is the only solution with 0 datum). Theorem 4.1 also proves that weak solutions may come to rest in finite time, a question posed in [150, pp. 88]. Lastly, note that the function $e(t)$ may be taken to be monotone decreasing so that rigidity fails for dissipative weak/mild solutions.

4.1.1. Outline of the proof of Theorem 4.1 and of intermittent convex integration schemes. The summary given here is similar to the one the authors gave in 22 , Section 4.2]. For clarity of the presentation, we omit any discussion of the energy profile $e(t)$, and refer the reader to [23] and [22, Section 7] for details.

The structure of the proof resembles that of Nash-type convex integration schemes, as described in Section 3.3.1. In order to construct weak solutions of (2.1) we proceed inductively, and for every $q \in \mathbb{N}_{0}$ we construct a solution $\left(v_{q}, \stackrel{\circ}{R}_{q}\right)$ to the Navier-Stokes-Reynolds system,

$$
\begin{aligned}
\partial_{t} v_{q}+\operatorname{div}\left(v_{q} \otimes v_{q}\right)+\nabla p_{q}-\nu \Delta v_{q} & =\operatorname{div} \stackrel{\circ}{R}_{q}, \\
\operatorname{div} v_{q} & =0,
\end{aligned}
$$

where the stress $\stackrel{\circ}{R}_{q}$ is traceless symmetric, and $p_{q}=(-\Delta)^{-1} \operatorname{div} \operatorname{div}\left(v_{q} \otimes v_{q}-\stackrel{\circ}{R}_{q}\right)$. 
As in Section 3.3.1 we work with a superexponentially growing sequence of frequencies $\lambda_{q}$ which obeys $\lambda_{q+1} \approx \lambda_{q}^{b}$ and $\lambda_{0} \gg 1$, except that this time $b \gg 1$, so we think of the frequency $\lambda_{q+1}$ as being much much larger than $\lambda_{q}$. Moreover, as in (3.3), we denote the velocity increment by $v_{q+1}-v_{q}=w_{q+1}$, and we heuristically view $w_{q+1}$ as a function whose Fourier support lies inside a ball of radius proportional to $\lambda_{q+1}$ around the origin.

From experience with $3 \mathrm{D}$ Euler, we expect that $\mathbb{P}_{\lesssim \lambda_{q}}\left(w_{q+1} \otimes w_{q+1}\right)+\stackrel{\circ}{R}_{q}$ has to be made as small as possible, so that if we wish the sequence of approximate velocities $v_{q}=v_{0}+\sum_{q^{\prime}<q} w_{q^{\prime}}$ to converge strongly in $C_{t}^{0} L_{x}^{2}$ as $q \rightarrow \infty$ to a weak solution of the Navier-Stokes equations, then the sequence $\left(v_{q}, \stackrel{\circ}{R}_{q}\right)$ has to be constructed such that $\stackrel{\circ}{R}_{q}$ vanishes in $C_{t}^{0} L_{x}^{1}$ as $q \rightarrow \infty$. This is a major difference when compared to the uniform in space convex integration used for the Euler equations: here Reynolds stresses (errors) are measured in $L_{x}^{1}$, whereas velocity increments and approximate velocities are measured in $L_{x}^{2}$; this is essentially because $\stackrel{\circ}{R}_{q}$ is quadratic in $w_{q+1}$. Thus, in analogy to (3.7) and (3.8), the inductive scheme is designed to propagate the bounds

$$
\begin{aligned}
\left\|w_{q+1}\right\|_{C_{t}^{0} L_{x}^{2}} & \leq \lambda_{q+1}^{-\beta}, \\
\left\|\stackrel{\circ}{R}_{q}\right\|_{C_{t}^{0} L_{x}^{1}} & \leq \lambda_{q+1}^{-2 \beta},
\end{aligned}
$$

for some $\beta>0$, which will be taken to be very small (in terms of the parameter $b$ in $\lambda_{q+1} \approx \lambda_{q}^{b}$ ).

In order to streamline the notation, for the remainder of this section we omit the $C_{t}^{0}$ from all norms and simply denote $\|\cdot\|_{C_{t}^{0} B_{x}}$ as $\|\cdot\|_{B}$ for any Banach space $B$, because all norms are taken to be uniform in time. From the bound (4.2a) and our heuristic about the Fourier support of $w_{q+1}$, we may expect that

$$
\left\|w_{q+1}\right\|_{\dot{H}^{s}} \lesssim \lambda_{q+1}^{s-\beta}
$$

which is suggestive of the fact that $v_{q} \rightarrow v=v_{0}+\sum_{q>0} w_{q}$ strongly in $C_{t}^{0} H_{x}^{\beta^{\prime}}$ for $\beta^{\prime}<\beta$. Moreover, viewing the momentum equation in (4.1) as a forced Stokes system, from the maximal regularity of the Stokes equation we deduce that

$$
\left\|\nabla v_{q}\right\|_{L^{p}} \lesssim\left\|v_{q} \otimes v_{q}\right\|_{L^{p}}+\left\|\stackrel{\circ}{R}_{q}\right\|_{L^{p}} \lesssim\left\|v_{q}\right\|_{L^{2 p}}^{2}+\left\|\stackrel{\circ}{R}_{q}\right\|_{L^{p}},
$$

with bounds that degenerate as $\nu \rightarrow 0$. Thus, letting $p>1$ be sufficiently close to 1 , we may deduce that $\nabla v_{q} \rightarrow \nabla v$ strongly in $C_{t}^{0} L_{x}^{1+\beta^{\prime \prime}}$ for some $0<\beta^{\prime \prime}<\beta^{\prime}$. Thus, using (4.2a) and (4.2b) the regularity of the limiting weak solution $v$ which we claimed in Theorem 4.1 follows, except that $\beta$ is replaced with $\beta^{\prime \prime}>0$ (the reader should not be confused by the fact that the $\beta$ in Theorem 4.1 is not the same parameter as the one in (4.2a) and (4.2b) ).

Subtracting the equations (4.1) at level $q+1$ and level $q$, similarly to (3.4), we obtain

$$
\begin{aligned}
\operatorname{div} \stackrel{\circ}{R}_{q+1}-\nabla\left(p_{q+1}-p_{q}\right)= & \operatorname{div}\left(w_{q+1} \otimes w_{q+1}+\stackrel{\circ}{R}_{q}\right)+\partial_{t} w_{q+1}-\nu \Delta w_{q+1} \\
& +\operatorname{div}\left(v_{q} \otimes w_{q+1}+w_{q+1} \otimes v_{q}\right) .
\end{aligned}
$$

In order to cancel the previous stress $\stackrel{\circ}{R}_{q}$ in the first term on the right side of (4.3), as in the proof of Theorem 3.3, the principal part of perturbation, $w_{q+1}^{(p)}$, is taken 
to be of the form (3.5), where the coefficient functions $a_{\xi}\left(\stackrel{\circ}{R}_{q}\right)$ are chosen to satisfy the low mode cancellation identity (3.6).

The main difficultly in implementing a convex integration scheme for the NavierStokes equations is ensuring that the dissipative term $\nu \Delta w_{q+1}$ on the right side of (4.3) can be treated as an error in comparison to the quadratic term $\operatorname{div}\left(w_{q+1} \otimes w_{q+1}\right)$. Note that the building blocks $W_{\xi}$ from the Euler construction (in conjunction with the ansatz (3.5)) do not have this desirable property, as they satisfy the heuristic pointwise in space bounds $\nu \Delta w_{q+1} \approx \nu \lambda_{q+1}^{2-\beta}$ and $\operatorname{div}\left(w_{q+1} \otimes w_{q+1}\right) \approx \lambda_{q+1}^{1-2 \beta}$ (recall, $0<\beta \ll 1$ and $\left.\lambda_{q+1} \gg 1\right)$.

The fundamental difference that the intermittent convex integration scheme presents over the Euler $L_{t, x}^{\infty}$ and $C_{t, x}^{\alpha}$ schemes is that the building blocks $W_{\xi}$ are chosen to be intermittent, by which we mean that the size of their $L^{p}$ norms differs vastly for different values of $p$. In particular, in view of (4.2a) and 4.2b) it is natural to normalize

$$
\left\|W_{\xi}\right\|_{L^{2}} \approx 1
$$

and in order to control the term stress arising from $\nu \Delta w_{q+1}$, it will immediately become apparent that we need to ensure

$$
\left\|W_{\xi}\right\|_{L^{1}} \approx \lambda_{q+1}^{-1-\epsilon}
$$

for some $\epsilon>0$. To see this, we use the -1 order linear operator $\mathcal{R}$ which inverts the divergence, and heuristically estimate the contribution of the dissipative term resulting from the principal perturbation $\nu \Delta w_{q+1}^{(p)}$ to the Reynolds stress error $\stackrel{\circ}{R}_{q+1}$ :

$$
\begin{aligned}
\left\|\mathcal{R}\left(\nu \Delta w_{q+1}^{(p)}\right)\right\|_{L^{1}} & \lesssim \nu \sum_{\xi}\left\|\nabla a_{\xi}\left(\stackrel{\circ}{R}_{q}\right)\right\|_{L^{\infty}}\left\|W_{\xi}\right\|_{L^{1}}+\left\|a_{\xi}\left(\stackrel{\circ}{R}_{q}\right)\right\|_{L^{\infty}}\left\|\nabla W_{\xi}\right\|_{L^{1}} \\
& \lesssim \nu \lambda_{q+1}\left\|R_{q}\right\|_{L^{\infty}}^{1 / 2}\left\|W_{\xi}\right\|_{L^{1}} \\
& \lesssim \nu \lambda_{q+1}\left(\lambda_{q}^{2} \lambda_{q+1}^{-\beta}\right)\left\|W_{\xi}\right\|_{L^{1}},
\end{aligned}
$$

where we have used the heuristic that $\stackrel{\circ}{R}_{q}$ only contains frequencies $\lesssim \lambda_{q}$, whereas $W_{\xi}$ has frequencies as large as $\lambda_{q+1}$, and in the last inequality used (4.2b) and the Sobolev embedding $W^{4,1} \subset L^{\infty}$. In order to ensure that the right side of (4.6) produces an error which is compatible with the inductive stress assumption (4.2b) at level $q+1$, and taking into account that $\nu \approx 1$, since $\lambda_{q+1} \approx \lambda_{q}^{b}$, we must ensure that

$$
\lambda_{q+1}\left(\lambda_{q}^{2} \lambda_{q+1}^{-\beta}\right)\left\|W_{\xi}\right\|_{L^{1}} \ll \lambda_{q+2}^{-2 \beta} \quad \Leftrightarrow \quad\left\|W_{\xi}\right\|_{L^{1}} \ll \lambda_{q+1}^{-1-2 \beta b+\beta-\frac{2}{b}} .
$$

The inequality (4.7) justifies the need to ensure (4.5). The former condition is, roughly speaking, also sufficient: first take $b \gg 1$ and then $\beta \ll 1 / b$, in terms of the $\epsilon$ in (4.5).

The bounds (4.4) and (4.5) justify the need to have building blocks $W_{\xi}$ which are intermittent. In Fourier space, this means that their Fourier support is spread out (think, a Dirichlet kernel vs a plane wave), whereas in physical space this means that the supports have very small measure, but that the $W_{\xi}$ have very high amplitude on this support. We also note that in $n$ dimensions, with the normalization (4.4), based on a simple scaling argument one may deduce that the smallest the $L^{1}$ norm of $W_{\xi}$ can be made is $\lambda_{q+1}^{-n / 2}$. In view of the requirement (4.5), this shows that in the two dimensional case $n=2$, the proof breaks down, as it should. Moreover, 
this heuristic shows that the higher the dimension $n$ is, the easier it is to achieve (4.5).

With the above requirements in mind, intermittent Beltrami waves were introduced 23 to serve as new $W_{\xi}$ 's. These waves modify the usual Beltrami plane waves used in the Euler constructions, by adding oscillations that mimic the structure of a three dimensional Dirichlet kernel, and they are compactly supported in Fourier space. Based on an analogy with Mikado flows [51, in the joint work with Colombo 16 intermittent jets were introduced to serve as $W_{\xi}$ 's. These flows achieve the same level of concentration in terms of (4.5) as intermittent Beltrami waves, but have a number of advantageous properties since instead of being compactly supported in Fourier space, they are compactly supported in physical space, and as such may be chosen to satisfy $\operatorname{supp} W_{\xi} \otimes W_{\xi^{\prime}}=\emptyset$ for $\xi \neq \xi^{\prime}$. This reduces the number of error terms which have to be estimated on the right side of (4.3). Besides having a different scale of periodization and concentration (cf. the estimate (4.9) below), one of the major differences between intermittent jets and Mikado flows is that intermittent jets are not anymore stationary solutions of the 3D Euler equations (neither were the intermittent Beltrami waves). More precisely, in order to obtain (4.5) with $\epsilon>0$, we need to (slowly) cut the pipe flows in the direction parallel to the pipe (in addition to the usual cutoff in the direction orthogonal to the pipe). This turns out to cause a number of additional difficulties, as explained in (4.13) below.

For the case of intermittent jets, in order to parameterize the concentration of the $W_{\xi}$, we introduce two parameters $r_{\|}$(a length in the direction parallel to the pipe) and $r_{\perp}$ (a length in the direction perpendicular to the pipe) such that

$$
\frac{\lambda_{q}}{\lambda_{q+1}} \ll r_{\perp} \ll r_{\|} \ll 1
$$

Each intermittent jet $W_{\xi}$ is defined to be supported on $\approx\left(r_{\perp} \lambda_{q+1}\right)^{3}$ many cylinders of diameter $\approx \frac{1}{\lambda_{q+1}}$ and length $\approx \frac{r_{\|}}{r_{\perp} \lambda_{q+1}}$. In particular, the measure of the support of $W_{\xi}$ is $\approx r_{\|} r_{\perp}^{2}$. We note that such scalings are consistent with the jet $W_{\xi}$ being of frequency $\approx \lambda_{q+1}$. Finally, we normalize $W_{\xi}$ such that its $L^{2}$ norm is $\approx 1$. Hence by scaling arguments, one expects an estimate of the form

$$
\left\|W_{\xi}\right\|_{W^{N, p}} \lesssim r_{\perp}^{2 / p-1} r_{\|}^{1 / p-1 / 2} \lambda_{q+1}^{N}
$$

for $1 \leq p \leq \infty$ and $N \geq 0$. In particular, the smallness condition (4.5) holds once we require that

$$
r_{\perp} r_{\|}^{1 / 2} \approx \lambda_{q+1}^{-1-\epsilon}
$$

for some $\epsilon \in(0,1 / 2)$. Note that (4.10), together with the condition (4.8), rules out geometric growth of the frequency $\lambda_{q}$, i.e., $b \gg 1$.

Next, consider the estimate (4.2a). Using Hölder's inequality to naïvely estimate the $L^{2}$ norm of the principal perturbation $w_{q+1}^{(p)}$, we obtain

$$
\left\|w_{q+1}^{(p)}\right\|_{L^{2}} \lesssim \sum_{\xi}\left\|a_{\xi}\left(\stackrel{\circ}{R}_{q}\right)\right\|_{L^{\infty}}\left\|W_{\xi}\right\|_{L^{2}} \lesssim\left\|\stackrel{\circ}{R}_{q}\right\|_{L^{\infty}}^{1 / 2} .
$$

In view of (4.9) with $p=\infty$, we cannot however inductively propagate good estimates on the $L^{\infty}$ norm of $\stackrel{\circ}{R}_{q}$, and as such, the above naïve estimate is not suitable 
in order to obtain (4.2a). To obtain a better estimate, we will utilize the following heuristic observation: given a function $f$ with frequency contained in a ball of radius $\lambda$ and a $(\mathbb{T} / \kappa)^{3}$-periodic function $g$, if $\kappa \gg \lambda$, then

$$
\|f g\|_{L^{p}} \lesssim\|f\|_{L^{p}}\|g\|_{L^{p}}
$$

A precise statement for the above heuristic is given in [23. Lemma 3.7]. Hence using that $\stackrel{\circ}{R}_{q}$ is of frequency roughly $\lambda_{q}$, and that $W_{\xi}$ is $(\mathbb{T} / \kappa)^{3}$-periodic with $\kappa=\lambda_{q+1} r_{\perp}$, and since by (4.8) we have $\lambda_{q+1} r_{\perp} \gg \lambda_{q}$, we obtain from (4.11) that

$$
\left\|w_{q+1}^{(p)}\right\|_{L^{2}} \lesssim \sum_{\xi}\left\|a_{\xi}\left(\stackrel{\circ}{R}_{q}\right)\right\|_{L^{2}}\left\|W_{\xi}\right\|_{L^{2}} \lesssim\left\|\stackrel{\circ}{R}_{q}\right\|_{L^{1}}^{1 / 2} \lesssim \lambda_{q+1}^{-\beta},
$$

where we have used (4.2b). This proves the feasibility of (4.2a), which is crucial if we wish to obtain a finite energy weak solution in the limit.

We return to discuss the remaining terms in (4.3). First, we note that the last term on the right side of (4.3) may be bounded quite easily, by using $v_{q}=$ $v_{0}+\sum_{q^{\prime} \leq q} w_{q}$, the Sobolev embedding $H^{2} \subset L^{\infty}$, and an argument similar to (4.12) but in $L^{1}$ :

$$
\begin{aligned}
\left\|w_{q+1}^{(p)} \otimes v_{q}\right\|_{L^{1}} & \lesssim\left\|v_{q}\right\|_{L^{\infty}} \sum_{\xi}\left\|a_{\xi}\left(\stackrel{\circ}{R}_{q}\right)\right\|_{L^{1}}\left\|W_{\xi}\right\|_{L^{1}} \\
& \lesssim\left(\lambda_{q}^{2}\left\|v_{q}\right\|_{L^{2}}\right) \sum_{\xi}\left\|a_{\xi}\left(\stackrel{\circ}{R}_{q}\right)\right\|_{L^{2}}\left\|W_{\xi}\right\|_{L^{1}} \\
& \lesssim \lambda_{q+1}^{2 / b} \lambda_{q+1}^{-\beta} \lambda_{q+1}^{-1-\beta}
\end{aligned}
$$

where we have used (4.2a), (4.2b), and (4.5). Since $b \gg 1$ and $\beta \ll 1 / b$, the above estimate is easily seen to be $\ll \lambda_{q+2}^{-2 \beta}$, as is required by (4.2b) at level $q+1$.

The only terms from (4.3) are the high frequency part of the oscillation error, and the temporal derivative term. We note crucially that in comparison to the Beltrami or the Mikado waves used for the 3D Euler constructions, the intermittent building blocks used in [16,23. introduce addition difficulties in handling the oscillation error, because the intermittent building blocks do not anymore solve stationary 3D Euler (to leading order). For the intermittent jets of [16] we have

$$
\operatorname{div}\left(w_{q+1}^{(p)} \otimes w_{q+1}^{(p)}+\stackrel{\circ}{R}_{q}\right) \approx \sum_{\xi} 2 a_{\xi}^{2} W_{\xi} \cdot \nabla W_{\xi}+(\text { high frequency error })
$$

Similar to how the Nash error for the Euler equations was dealt with (see (3.11)), the high frequency error in (4.13) experiences a gain when one inverts the divergence equation. Note however that this high frequency is not $\lambda_{q+1}$ since the lowest active frequency in $W_{\xi} \otimes W_{\xi}-f_{\mathbb{T}^{3}} W_{\xi} \otimes W_{\xi}$ is $\approx \lambda_{q+1} r_{\perp}$; nonetheless, $\lambda_{q+1} r_{\perp} \gg \lambda_{q}$ as in (4.8).

In order to take care of the main term in (4.13), the intermittent jets are carefully designed to oscillate in time such that this term can be written as a temporal derivative

$$
\sum_{\xi} 2 W_{\xi} \cdot \nabla W_{\xi}=\frac{1}{\mu} \partial_{t}\left(\sum_{\xi}\left|W_{\xi}\right|^{2} \xi\right)
$$


for some large parameter $\mu$. This error can absorbed by introducing, in addition to the principal corrector, also a temporal corrector $w_{q+1}^{(t)}$ defined as

$$
w_{q+1}^{(t)}:=-\frac{1}{\mu} \mathbb{P}_{H} \mathbb{P}_{\neq 0}\left(\sum_{\xi} a_{\xi}^{2}\left(\stackrel{\circ}{R}_{q}\right)\left|W_{\xi}\right|^{2} \xi\right),
$$

where $\mathbb{P}_{H}$ is the Helmholtz projection and $\mathbb{P}_{\neq 0}$ is the projection onto functions with mean zero. Thus pairing the oscillation error with the time derivative of the temporal corrector, we obtain

$\operatorname{div}\left(w_{q+1}^{(p)} \otimes w_{q+1}^{(p)}+\stackrel{\circ}{R}_{q}\right)+\partial_{t} w_{q+1}^{(t)} \approx$ (pressure gradient) + (high frequency error).

This identity is crucial for the intermittent convex integration scheme for 3D NavierStokes to close. In essence, the intermittent building blocks we have chosen in the construction do not solve to leading order the stationary 3D Euler equations, but instead to leading order they solve the time-dependent 3D Euler equations.

Finally, analogous to the Euler case, we define a divergence corrector $w_{q+1}^{(c)}$ to fix the fact that $w_{q+1}^{(p)}$ is not, as defined, divergence free. The total perturbation $w_{q+1}$ is then defined to be

$$
w_{q+1}:=w_{q+1}^{(p)}+w_{q+1}^{(t)}+w_{q+1}^{(c)} .
$$

Due to $w_{q+1}^{(t)}$ and $w_{q+1}^{(c)}$, a number of new error terms arise on the right side of (4.3), most of them being benign. The main new error term arises from the temporal oscillation in the definition of the intermittent jets, which introduce an error from the term $\partial_{t} w_{q+1}^{(p)}$ which has a factor of $\mu$ in front. On the other hand, the oscillation error is inversely proportional to $\mu$, and thus one has to carefully choose $\mu$ to optimize between these two errors. We omit these technical details and refer the reader to the summary given in [22, Section 7].

4.1.2. Vanishing viscosity limits of finite energy mild solutions. A natural question to consider is whether the nonconservative weak solutions to the 3D Euler equations, which were obtained in [20,93, arise as vanishing viscosity limits of weak solutions to the Navier-Stokes equations. In this direction, as a direct consequence of the proof of Theorem 4.1, one obtains that the answer is yes, at least when one considers a sufficiently wide class of weak solutions to (2.1):

Theorem 4.2 (Theorem 1.3 in $[23]$ ). For $\bar{\beta}>0$ let $v \in C_{t, x}^{\bar{\beta}}\left(\mathbb{T}^{3} \times[-2 T, 2 T]\right.$ ) be a zero-mean weak solution of the $3 D$ Euler equations. Then there exists $\beta>0, a$ sequence $\nu_{n} \rightarrow 0$, and a uniformly bounded sequence $v^{\left(\nu_{n}\right)} \in C_{t}^{0}\left([0, T] ; H_{x}^{\beta}\left(\mathbb{T}^{3}\right)\right)$ of weak solutions to the Navier-Stokes equations in the sense of Definition 2.2, with $v^{\left(\nu_{n}\right)} \rightarrow v$ strongly in $C_{t}^{0}\left([0, T] ; L_{x}^{2}\left(\mathbb{T}^{3}\right)\right)$.

The above result shows that being a strong $C_{t}^{0} L_{x}^{2}$ limit of weak solutions to the Navier-Stokes equations, in the sense of Definition 2.2, cannot serve as a selection criterion for weak solutions of the Euler equation. Theorem 4.2 however does not rule out a selection criterion based on vanishing viscosity limits of Leray-Hopf weak solutions; we refer the reader to [5, 7, 8, 175] where this question is discussed in the context of convex integration, symmetry breaking, and in the presence of solid boundaries. 
4.2. Nonuniqueness of weak solutions with partial regularity in time. As mentioned above, intermittent jets were introduced in the joint work of the authors and Colombo [16. The main goal of that paper was to give an example of a mild/weak solution to the Navier-Stokes equation whose singular set of times $\Sigma_{T} \subset(0, T]$ is both nonempty and has Hausdorff dimension strictly less than 1 , i.e., it has partial regularity in time (a property that all Leray-Hopf weak solutions possess [115]). The main result in [16] is:

Theorem 4.3 (Theorem 1.1 in [16]). There exists $\beta>0$ such that the following holds. For $T>0$, let $u^{(1)}, u^{(2)} \in C^{0}\left([0, T] ; \dot{H}^{3}\left(\mathbb{T}^{3}\right)\right)$ be two strong solutions of the Navier-Stokes equations with initial data $u^{(1)}(\cdot, 0)$ and $u^{(2)}(\cdot, 0)$ of zero mean. There exists a weak solution $v \in C^{0}\left([0, T] ; H^{\beta}\left(\mathbb{T}^{3}\right) \cap W^{1,1+\beta}\left(\mathbb{T}^{3}\right)\right)$ of (2.1), in the sense of Definition 2.2, such that

$$
v \equiv u^{(1)} \quad \text { on } \quad[0, T / 3], \quad \text { and } \quad v \equiv u^{(2)} \quad \text { on } \quad[2 T / 3, T] .
$$

Moreover, for every such $v$ there exists a zero Lebesgue measure set of times $\Sigma_{T} \subset$ $(0, T]$ with Hausdorff dimension less than $1-\beta$, such that

$$
v \in C^{\infty}\left(\left((0, T] \backslash \Sigma_{T}\right) \times \mathbb{T}^{3}\right) .
$$

In particular, the weak solution $v$ is almost everywhere smooth.

Note that Theorem 4.3 establishes the nonuniqueness of weak/mild solutions (cf. Defintion 2.2) to the Cauchy problem for the 3D Navier-Stokes equation, for any initial datum which permits the local-in-time solvability of (4.3) (e.g., $v_{0} \in \dot{H}^{1 / 2}$ or $\left.v_{0} \in L^{3}\right)$. Indeed, for such $v_{0}$ one may uniquely define the solution $u^{(1)}$ in Theorem 4.3 for a suitable time $T$, and then all one needs to do is choose $u^{(2)}$ as a shear flow whose kinetic at time $T$ is larger than that of $v_{0}$. Due to the energy inequality, the weak solution provided by Theorem 4.3 is not the same as the global-in-time Leray-Hopf weak solution with datum $v_{0}$, yielding nonuniqueness. In fact, this argument may be modified to hold for any incompressible $v_{0} \in L^{2}$, cf. [16, Remark 1.4].

The proof of Theorem 4.3 builds on the one of Theorem 4.1. but additionally it inductively keeps track of good time intervals on which the approximate solutions $v_{q}$ are in fact smooth solutions of (2.1) and are untouched in later inductive steps. This is achieved by employing the method of gluing introduced in 20, 93. Taking the countable union of the good regions over each inductive step $q$ a fractal set is formed; on this set the solution is $C^{\infty}$ smooth, and the complement of this set has Hausdorff dimension strictly less than 1 .

4.3. Further developments of intermittent convex integration schemes. The new flavor of convex integration introduced in [23] and [16], has recently been extended and improved, in order to obtain nonuniqueness and other flexibilitytype results for various models arising in hydrodynamics and PDEs in general. We mention here a few of these results, as they present interesting applications of the idea that flexibility may also be attained via low integrability, not just low regularity.

- The transport equation. Using a version of the classical Mikado flows but which also take into account spatial concentrations (called intermittent Mikado flows), the authors of [128-130] have established the existence of nonrenormalized solutions, as well as Eulerian nonuniqueness, for the 
transport and continuity equations with Sobolev vector fields. These striking counterexamples point towards the sharpness of the classical results for renormalized solutions 65. We also refer the reader to [14] where a number of new results (both in terms of uniqueness and nonuniqueness) were obtained for positive solutions to the transport equation, and to 34] where temporal intermittency and oscillation is introduced in the intermittent convex integration scheme in order to extend the integrability range.

- Hyperviscous 3D Navier-Stokes. The authors of [122] and [16] have independently proven that intermittent convex integration is also applicable to establish the nonuniqueness of finite energy weak/mild solutions to the fractionally dissipative 3D Navier-Stokes equations with dissipation $(-\Delta)^{\alpha}$, and $\alpha<5 / 4$ (the so-called Lions criticality threshold [117]). We note that similar results may also be established [121] for the hypoviscous 2D NavierStokes equation $\alpha<1$.

- The stochastic 3D Navier-Stokes equations. Nonuniqueness in law for the stochastic 3D Navier-Stokes system, with either an additive or a linear multiplicative noise driven by a Wiener process, was recently obtained in a remarkable paper [88]. See also the result [179] which considers the stochastic fractionally dissipative Navier-Stokes equation in the full supercritical regime $\alpha<5 / 4$.

- Other hydrodynamic models. In [48, it is shown that intermittent convex integration methods can be adapted to prove the nonuniqueness of Leray-Hopf weak solutions for the 3D Hall-MHD system. We note that the nonuniqueness result in 48 fundamentally relies on the presence of the Hall term curk (curk $B \times B)$ which is dominant when compared to $\Delta B$ (see also the recent result [49] for the electron-MHD system). Lastly, we mention that the technique of convex integration was applied to obtain nonuniqueness of distributional solutions for a model of non-Newtonian fluids called power law fluids [24].

4.4. Some open problems in the context of 3D Navier-Stokes. To date it remains open to show that the regularity parameter $\beta$ in Theorems 4.1 or 4.3 may be taken to be "significant", for instance $\leq 1 / 9$. Due to [83, the results cannot hold for $\beta \geq 1 / 2$. Note however that one may formally write the Navier-Stokes system in arbitrary dimensions $n \geq 2$, and that in higher dimensions we have stronger forms of intermittency/spatial concentration. Exploring this observation, in 123 . it was shown that for $n=4$, the statement corresponding to Theorem 4.1 for timeindependent solutions holds for $\beta<1 / 200$. Moreover, in [164, Theorem 29], it is shown that as we send the dimension $n \rightarrow \infty$, one may prove Theorem 4.1 with $\beta \rightarrow 1 / 2^{-}$.

Equally challenging to increasing the regularity of the solutions in Theorems 4.1 or 4.3 seems to be to improve their integrability from $C_{t}^{0} L_{x}^{2}$ to $C_{t}^{0} L_{x}^{p}$ for some $p \in[2,3)$ which "significantly" departs from $p=2$. Note that due to [84] we know that uniqueness holds for mild solutions in $C_{t}^{0} L_{x}^{p}$ with $p \geq 3$; establishing the sharpness of this criterion via convex integration appears to be out of reach with current methods; see [22, Problem 8]. 
The energy inequality (2.5) presents a formidable obstacle towards extending the results of Theorems 4.1 or 4.3 to the class of Leray-Hopf weak solutions. However, what happens if we give up on the energy equality and only retain the regularity/integrability provided by the energy class? Recall the discussion in Section 2.1.2. The weak/mild solutions of Definition 2.2 need not satisfy the energy inequality (2.5) even if they lie in the Leray-Hopf class $C_{\text {weak }, t}^{0} L_{x}^{2} \cap L_{x}^{2} \dot{H}_{x}^{1}$, as long as they do not belong to $L_{t, x}^{4}$ or another space with similar scaling [151]. This leaves open an intriguing possibility:

Problem 4.4 (Nonuniqueness of mild solutions in the energy class). Is it possible to prove a stronger version of Theorem 4.1, by additionally requiring that $v \in$ $L^{2}\left((0, T) ; \dot{H}^{1}\left(\mathbb{T}^{3}\right)\right)$ ?

We again emphasize that Problem 4.4 does not require that the weak solution $v$ satisfies the energy inequality. Curiously, in terms of the parabolic scaling that is natural for the 3D Navier-Stokes system, $v(x, t) \mapsto v_{\lambda}(x, t)=\lambda v\left(\lambda x, \lambda^{2} t\right)$, the space $L_{t}^{2} \dot{H}_{x}^{1}$ scales in the same way as the space $L_{t}^{\infty} L_{x}^{2}$, which is already present in Theorem 4.1. To date, however, it is not known how to parabolically trade temporal integrability for spatial regularity within the framework of an intermittent convex integration scheme for 3D Navier-Stokes.

\section{Convex integration constructions for the MHD equations}

As discussed in Section 2.3.3 the rigidity parts (国) in Conjecture 2.10 and (国) in Conjecture 2.11 have been established rigorously. In this section, we discuss partial progress towards the flexible parts of these Onsager-type dichotomies for ideal MHD (2.19).

5.1. Bounded wild weak solutions of the MHD system. Concerning the flexible part (b) of Conjecture 2.10, we start with the example of Bronzi, Lopes Filho, and Nussenzveig Lopes [13]. The authors in [13] impose a symmetry assumption which embeds the ideal MHD system into a two-and-a-half dimensional Euler flow. If $v=\left(v_{1}, v_{2}, v_{3}\right)\left(x_{1}, x_{2}\right)$ is a weak solution of 3D Euler independent of $x_{3}$, then setting the velocity field in (2.19) to $\left(v_{1}, v_{2}, 0\right)$ and the magnetic field to $\left(0,0, v_{3}\right)$, we obtain a weak solution of the ideal MHD system, which is independent of $x_{3}$. If $v_{3} \not \equiv 0$, then such a weak solution has a nontrivial magnetic field. For $v$ as in part (b) of Theorem 2.4, the total energy $\mathcal{E}$ (defined in (2.21)) of these weak solutions is dissipated, but both $\mathcal{H}_{\omega, B}=\mathcal{H}_{B, B}=0$, and are hence constant functions of time (recall the definitions (2.26) and (2.27)). Thus, the additional flexibility requirement of Conjecture 2.10 that $\mathcal{H}_{\omega, B}$ is not conserved-seems to cause new difficulties when compared to the Euler case.

A more fundamental source of difficulties present in the analysis of the ideal MHD system is that as soon as $(v, B) \in L_{t, x}^{3}$, the magnetic helicity $\mathcal{H}_{B, B}$ must be a constant function of time [1,76, 100. But typically, convex integration schemes are able to "break" all quadratic conservation laws which are well defined at the regularity level of the weak solutions constructed. Thus, it is very nontrivial to construct solutions which conserve the magnetic helicity but do not conserve the Elsässer energies 14

\footnotetext{
${ }^{14}$ One is faced with a similar difficulty in attacking Conjecture 2.7 or in trying to establish flexibility of the Casimirs for 2D SQG or 2D Euler; see Remark 2.9.
} 
The first such result in the context of bounded weak solutions was recently obtained by Faraco, Lindberg, and Székelyhidi 78 , who use the $L_{t, x}^{\infty}$ convex integration scheme of [55] to construct bounded weak solutions of (2.19) which have compact support in space and time. The authors of [78] also establish a number of rigidity results for $2 \mathrm{D}$ ideal $\mathrm{MHD}$, but in terms of flexibility, their main conclusion is the $3 \mathrm{D}$ result:

Theorem 5.1 (Theorem 1.1 in [78]). There exist bounded, compactly supported weak solutions of ideal $M H D$ in $\mathbb{R}^{3}$, with both $v, B$ nontrivial, such that neither total energy nor cross helicity is conserved in time.

Since the solutions constructed in Theorem 5.1 have compact support in time, and since they conserve the magnetic helicity, we have that $\mathcal{H}_{B, B}(t)$ must vanish at all times $t \in \mathbb{R}$, even though $B$ is not identically equal to zero.

Theorem 5.1 represents the first result towards the flexible part (b) of Conjecture 2.10. The analogous result in the Onsager program for 3D Euler would be Theorem 3.1 from [55].

The proof of Theorem 5.1 uses the classical framework provided by the $L_{t, x}^{\infty}$ flavor of convex integration of De Lellis and Székelyhidi [55] and the Tartar framework [167, as axiomatized in [163. Broadly speaking, the additional rigidity provided by the conservation of magnetic helicity is a manifestation of the weakly closed nature of the Maxwell equations for the magnetic field [166. As such, when performing the plane-wave analysis, the interaction with the momentum equation for the velocity field $v$, which has a large relaxation, has to be considered very carefully. For instance, in an earlier work [76] Faraco and Lindberg show that there exist nontrival smooth strict subsolutions of 3D ideal MHD, with compact support in space-time, but that the interior of the $3 \mathrm{D} \Lambda$-convex hull is empty, which makes it difficult to implement a convex integration scheme starting from this subsolution. The authors of 78 instead develop a variant of the convex integration scheme directly on differential two-forms, retaining consistency with the phase space geometry of the 3D ideal MHD system; see [78, Sections 3, 4] for details.

5.2. Weak solutions which do not conserve magnetic helicity. In order to make progress on the flexible part (b) of Conjecture 2.11, one has to be able to construct weak solutions of ideal MHD (2.19) which have finite total energy (as required by Defintion 2.8), but do not lie in $L_{t, x}^{3}$, since otherwise they would conserve $\mathcal{H}_{B, B}$. Moreover, in view of Taylor's conjecture (cf. Theorem 2.13), such weak solutions cannot be constructed as weak ideal limits of MHD Leray-Hopf weak solutions (the usual weak-compactness methods via smooth approximations fail anyway, for the same reasons they fail in 3D Euler). Clearly, the uniform in space convex integration developed in the proof of Theorem 5.1] is not well suited to achieve this goal. However, the intermittent convex integration scheme developed in the context of 3D Navier-Stokes (see Section 4) stands a chance, because it is exactly designed to explore the low integrability of weak solutions, via a careful choice of intermittent building blocks. This idea was explored by the authors and Beekie in [10]:

Theorem 5.2 (Theorem 1.4 in [10]). There exists $\beta>0$ such that the following holds. There exist weak solutions $(v, B) \in C\left([0,1], H^{\beta}\right)$ of (2.19), in the sense of Definition 2.8, which do not conserve magnetic helicity. In particular, there exist solutions as above with $2\left|\mathcal{H}_{B, B}(0)\right| \leq \mathcal{H}_{B, B}(1)$ and $\mathcal{H}_{B, B}(1)>0$. For these 
solutions the total energy $\mathcal{E}$ and cross helicity $\mathcal{H}_{\omega, B}$ are nontrivial, nonconstant functions of time.

Theorem 5.2 provides the first result on the flexible part (b) of Conjecture 2.11 by providing an example of a nonconservative weak solution to the ideal MHD equations which lies in $C_{t}^{0} L_{x}^{p}$ for some $p>2$, for which $\mathcal{E}, \mathcal{H}_{\omega, B}$ and $\mathcal{H}_{B, B}$ are all nontrivial. Besides establishing the nonuniqueness of weak solutions to (2.19) in the sense of Definition 2.8. Theorem 5.2 also gives an existence result for weak solutions to (2.19) at this low integrability level. Lastly, we emphasize that in view of Theorem 2.13, the weak solutions of 3D ideal MHD which we construct in Theorem 5.2 cannot be obtained as weak ideal limits from Leray-Hopf weak solutions to (2.20).

The proof of Theorem 5.2 builds on the intermittent convex integration schemes developed in 16, 23. The main new difficulties arise from the specific geometric structure of the nonlinear terms in 3D MHD so that the intermittent building blocks used for 3D Navier-Stokes (intermittent Beltrami flows, intermittent jets, viscous eddies) are not useful for the ideal MHD system.

Informally, the building blocks used in the 3D Navier-Stokes proof are designed to handle the dissipative term $\Delta v$, and as such require more than two-dimensional intermittency $(\epsilon>0$ in (4.5) $)$; moreover, the high-frequency component of the oscillation error $\operatorname{div}(v \otimes v)$ is handled by introducing a temporal corrector. For ideal MHD, we do not have to worry about a dissipative term, and so the role of intermittency is different. Here intermittency is needed in order to treat the high frequency nonlinear oscillation errors arising from $\operatorname{div}(v \otimes v-B \otimes B)$ and $\operatorname{div}(v \otimes B-$ $B \otimes v)$, which cannot be fixed anymore by adding temporal correctors, essentially because the velocity intermittent building blocks $W_{\xi}$ and the magnetic intermittent building blocks $D_{\xi^{\prime}}$ have nontrivial overlap even if $\xi \neq \xi^{\prime}$. To see this, we first note that the structure of the MHD nonlinearities requires the building blocks' direction of oscillation, $\xi$, to be orthogonal to two direction vectors $\xi_{1}$ and $\xi_{2}$. These two orthogonal direction vectors are needed in order to simultaneously cancel both the previous velocity Reynolds stress, which is symmetric, and the previous magnetic stress, which is antisymmetric. This only permits the usage of one-dimensional intermittency in our building blocks, meaning the maximal smallness that can be gained in $\left\|W_{\xi}\right\|_{L^{1}}\left\|W_{\xi}\right\|_{L^{2}}^{-1}$ and $\left\|D_{\xi}\right\|_{L^{1}}\left\|D_{\xi}\right\|_{L^{2}}^{-1}$ is proportional to $\lambda_{q+1}^{-1 / 2}$ (compare to (4.4) and (4.5) for Navier-Stokes). This amount of gain is by itself not sufficient to close the scheme.

The main idea in the proof of Theorem 5.2 is to construct a set of intermittent building blocks adapted to the MHD geometry, which we call intermittent shear velocity flows $W_{\xi}$ and intermittent shear magnetic flows $D_{\xi}$. Their spatial support is given by thickened planes spanned by the two orthogonal vectors $\xi_{1}$ and $\xi_{2}$, their support has volume $\approx r_{\perp}$ (which plays a role akin to the $r_{\perp}$ in (4.8)); they are periodized to scale $\lambda_{q+1} r_{\perp}$, and their only direction of oscillation is given only by the vector $\xi$ which is orthogonal to both $\xi_{1}$ and $\xi_{2}$. Using these orthogonality properties, the contributions to the oscillation errors from $\xi=\xi^{\prime}$ can be handled suitably. In order to treat $\xi \neq \xi^{\prime}$, we note that the product of two rationally skew-oriented 1D intermittent building blocks is more intermittent than each one of them; it has two dimensional intermittency because the intersection of two thickened (nonparallel) planes is given by a thickened line, which has $2 \mathrm{D}$ smallness. That is, we may show that the Lebesgue measure of $\operatorname{supp} W_{\xi} \otimes W_{\xi}^{\prime}$, supp $D_{\xi} \otimes W_{\xi}^{\prime}$, and $\operatorname{supp} D_{\xi} \otimes D_{\xi}^{\prime}$ is $\approx r_{\perp}^{2}$ 
when $\xi \neq \xi^{\prime}$. Suitably choosing $r_{\perp}$ allows one to treat the remaining oscillation errors. In summary, intermittency is used in Theorem 5.2 to treat nonlinear errors, instead of linear ones for Navier-Stokes; we refer to [10] for details.

5.3. Some open problems in the context of the MHD system. The applicability of convex integration methods to the ideal MHD system is at an early development stage. For instance, although the authors of [78] have recently implemented a $L_{t, x}^{\infty}$ convex integration scheme for (2.19), to date it remains open to implement a $C_{t, x}^{\alpha}$ Nash-type convex integration, for any $\alpha \geq 0$. As such, the flexible part (b) of Conjecture 2.10 remains open.

As is the case with the intermittent convex integration schemes for 3D NavierStokes, it seems that fundamentally new ideas are needed to substantially increase the value of $\beta$ in Theorem $[5.2$, or to increase the integrability index of the solutions from [10 from $L_{x}^{2}$ to $L_{x}^{p}$ with $p$ closer to 3 . Because of this, part (b) of Conjecture 2.11 remains widely open.

\section{ACKNOWLEDGMENTS}

The authors are grateful to Susan Friedlander for stimulating discussions and for fruitful suggestions in improving this article. We also thank Rajendra Beekie and Matthew Novack for helpful comments.

\section{ABOut THE AUTHORS}

Tristan Buckmaster is assistant professor of mathematics at Princeton University. He completed his $\mathrm{PhD}$ at the University of Leipzig/Max Planck Institute for Mathematics in the Sciences, Leipzig, Germany, in 2014. He then spent three years as a Courant Instructor at New York University and has subsequently joined Princeton University in 2017. His main area of interest is partial differential equations with a particular focus on equations related to hydrodynamics.

Vlad Vicol is professor of mathematics at New York University, the Courant Institute for Mathematical Sciences. He received his $\mathrm{PhD}$ from the University of Southern California in 2010. He taught at the University of Chicago and Princeton University before joining the faculty at the Courant Institute in 2018. His research focuses on the analysis of partial differential equations arising in fluid dynamics.

\section{REFERENCES}

[1] H. Aluie, Hydrodynamic and magnetohydrodynamic turbulence: Invariants, cascades, and locality, Ph.D. thesis, Johns Hopkins University, 2009.

[2] H. Aluie and G. L. Eyink, Scale locality of magnetohydrodynamic turbulence, Physical Review Letters 104 (2010), no. 8, 081101.

[3] F. Anselmet, Y. Gagne, E. J. Hopfinger, and R. A. Antonia, High-order velocity structure functions in turbulent shear flows, J. Fluid Mech. 140 (1984), 63-89.

[4] V. I. Arnold and B. A. Khesin, Topological methods in hydrodynamics, Applied Mathematical Sciences, vol. 125, Springer-Verlag, New York, 1998. MR 1612569

[5] C. Bardos, M. C. Lopes Filho, D. Niu, H. J. Nussenzveig Lopes, and E. S. Titi, Stability of two-dimensional viscous incompressible flows under three-dimensional perturbations and inviscid symmetry breaking, SIAM J. Math. Anal. 45 (2013), no. 3, 1871-1885, DOI 10.1137/120862569. MR.3068562

[6] C. Bardos, L. Sekelikhidi Jr., and È. Videmann, On the absence of uniqueness for the Euler equations: the effect of the boundary (Russian, with Russian summary), Uspekhi Mat. Nauk 69 (2014), no. 2(416), 3-22, DOI 10.1070/rm2014v069n02abeh004886; English transl., Russian Math. Surveys 69 (2014), no. 2, 189-207. MR3236935 
[7] C. Bardos, L. Sekelikhidi Jr., and È. Videmann, Non-uniqueness for the Euler equations: the effect of the boundary (Russian, with Russian summary), Uspekhi Mat. Nauk 69 (2014), no. 2(416), 3-22, DOI 10.1070/rm2014v069n02abeh004886; English transl., Russian Math. Surveys 69 (2014), no. 2, 189-207. MR3236935

[8] C. Bardos, E. S. Titi, and E. Wiedemann, The vanishing viscosity as a selection principle for the Euler equations: the case of $3 D$ shear flow (English, with English and French summaries), C. R. Math. Acad. Sci. Paris 350 (2012), no. 15-16, 757-760, DOI 10.1016/j.crma.2012.09.005. MR.2981348

[9] G. K. Batchelor, The theory of homogeneous turbulence, Cambridge Monographs on Mechanics and Applied Mathematics, Cambridge at the University Press, 1953. MR 0052268

[10] R. Beekie, T. Buckmaster, and V. Vicol, Weak solutions of ideal MHD which do not conserve magnetic helicity, Ann. PDE 6 (2020), no. 1, Paper No. 1, 40, DOI 10.1007/s40818-020-00761. MR4105741

[11] M. A. Berger, Introduction to magnetic helicity, Plasma Physics and Controlled Fusion 41 (1999), no. 12B, B167.

[12] D. Biskamp, Nonlinear magnetohydrodynamics, Cambridge Monographs on Plasma Physics, vol. 1, Cambridge University Press, Cambridge, 1993. MR.1250152

[13] A. C. Bronzi, M. C. Lopes Filho, and H. J. Nussenzveig Lopes, Wild solutions for 2D incompressible ideal flow with passive tracer, Commun. Math. Sci. 13 (2015), no. 5, 13331343, DOI 10.4310/CMS.2015.v13.n5.a12. MR3344429

[14] E. Brué, M. Colombo, and C. De Lellis, Positive solutions of transport equations and classical nonuniqueness of characteristic curves, arXiv preprint arXiv:2003.00539 (2020).

[15] T. Buckmaster, Onsager's conjecture almost everywhere in time, Comm. Math. Phys. 333 (2015), no. 3, 1175-1198, DOI 10.1007/s00220-014-2262-z. MR3302631

[16] T. Buckmaster, M. Colombo, and V. Vicol, Wild solutions of the Navier-Stokes equations whose singular sets in time have hausdorff dimension strictly less than 1 , Journal of the EMS. In press. (2020).

[17] T. Buckmaster, C. De Lellis, P. Isett, and L. Székelyhidi Jr., Anomalous dissipation for 1/5-Hölder Euler flows, Ann. of Math. (2) 182 (2015), no. 1, 127-172, DOI 10.4007/annals.2015.182.1.3. MR3374958

[18] T. Buckmaster, C. De Lellis, and L. Székelyhidi Jr., Dissipative Euler flows with Onsagercritical spatial regularity, Comm. Pure Appl. Math. 69 (2016), no. 9, 1613-1670, DOI 10.1002/cpa.21586. MR3530360

[19] T. Buckmaster, C. De Lellis, and L. Székelyhidi Jr., Transporting microstructure and dissipative Euler flows, arXiv preprint arXiv:1302.2815 (2013).

[20] T. Buckmaster, C. de Lellis, L. Székelyhidi Jr., and V. Vicol, Onsager's conjecture for admissible weak solutions, Comm. Pure Appl. Math. 72 (2019), no. 2, 229-274, DOI 10.1002/cpa.21781. MR3896021

[21] T. Buckmaster, S. Shkoller, and V. Vicol, Nonuniqueness of weak solutions to the SQG equation, Comm. Pure Appl. Math. 72 (2019), no. 9, 1809-1874, DOI 10.1002/cpa.21851. MR.3987721

[22] T. Buckmaster and V. Vicol, Convex integration and phenomenologies in turbulence, EMS Surv. Math. Sci. 6 (2019), no. 1, 173-263, DOI 10.4171/emss/34. MR4073888

[23] T. Buckmaster and V. Vicol, Nonuniqueness of weak solutions to the Navier-Stokes equation, Ann. of Math. 189 (2019), no. 1, 101-144.

[24] J. Burczak, S. Modena, and L. Székelyhidi Jr., Non uniqueness of power-law flows, arXiv preprint arXiv:2007.08011 (2020).

[25] L. Caffarelli, R. Kohn, and L. Nirenberg, Partial regularity of suitable weak solutions of the Navier-Stokes equations, Comm. Pure Appl. Math. 35 (1982), no. 6, 771-831, DOI 10.1002/cpa.3160350604. MR673830

[26] R. E. Caflisch, I. Klapper, and G. Steele, Remarks on singularities, dimension and energy dissipation for ideal hydrodynamics and MHD, Comm. Math. Phys. 184 (1997), no. 2, 443455, DOI 10.1007/s002200050067. MR1462753

[27] A. Castro, C. Córdoba, and D. Faraco, Mixing solutions for the Muskat problem, arXiv:1605.04822 (2016).

[28] J.-Y. Chemin, Perfect incompressible fluids, Oxford Lecture Series in Mathematics and its Applications, vol. 14, The Clarendon Press, Oxford University Press, New York, 1998. Translated from the 1995 French original by Isabelle Gallagher and Dragos Iftimie. MR.1688875 
[29] A. Cheskidov, P. Constantin, S. Friedlander, and R. Shvydkoy, Energy conservation and Onsager's conjecture for the Euler equations, Nonlinearity 21 (2008), no. 6, 1233-1252, DOI 10.1088/0951-7715/21/6/005. MR2422377

[30] A. Cheskidov, M. C. L. Filho, H. J. N. Lopes, and R. Shvydkoy, Energy conservation in two-dimensional incompressible ideal fluids, Comm. Math. Phys. 348 (2016), no. 1, 129-143, DOI 10.1007/s00220-016-2730-8. MR3551263

[31] A. Cheskidov, S. Friedlander, and N. Pavlović, Inviscid dyadic model of turbulence: the fixed point and Onsager's conjecture, J. Math. Phys. 48 (2007), no. 6, 065503, 16, DOI 10.1063/1.2395917. MR2337019

[32] A. Cheskidov, S. Friedlander, and R. Shvydkoy, On the energy equality for weak solutions of the 3D Navier-Stokes equations, Advances in mathematical fluid mechanics, Springer, Berlin, 2010, pp. 171-175, DOI 10.1007/978-3-642-04068-9_10. MR2665030

[33] A. Cheskidov and X. Luo, Energy equality for the Navier-Stokes equations in weak-in-time Onsager spaces, Nonlinearity 33 (2020), no. 4, 1388-1403, DOI 10.1088/1361-6544/ab60d3. MR.4072378

[34] A. Cheskidov and X. Luo, Nonuniqueness of weak solutions for the transport equation at critical space regularity, arXiv:2004.09538 (2020).

[35] A. Cheskidov and R. Shvydkoy, Euler equations and turbulence: analytical approach to intermittency, SIAM J. Math. Anal. 46 (2014), no. 1, 353-374, DOI 10.1137/120876447. MR 3152734

[36] E. Chiodaroli, A counterexample to well-posedness of entropy solutions to the compressible Euler system, J. Hyperbolic Differ. Equ. 11 (2014), no. 3, 493-519, DOI 10.1142/S0219891614500143. MR.3261301

[37] E. Chiodaroli, C. De Lellis, and O. Kreml, Global ill-posedness of the isentropic system of gas dynamics, Comm. Pure Appl. Math. 68 (2015), no. 7, 1157-1190, DOI 10.1002/cpa.21537. MR 3352460

[38] E. Chiodaroli, E. Feireisl, and O. Kreml, On the weak solutions to the equations of a compressible heat conducting gas, Ann. Inst. H. Poincaré Anal. Non Linéaire 32 (2015), no. 1, 225-243, DOI 10.1016/j.anihpc.2013.11.005. MR3303948

[39] A. Choffrut, h-principles for the incompressible Euler equations, Arch. Ration. Mech. Anal. 210 (2013), no. 1, 133-163, DOI 10.1007/s00205-013-0639-3. MR3073150

[40] M. Colombo, C. De Lellis, and L. De Rosa, Ill-posedness of Leray solutions for the hypodissipative Navier-Stokes equations, Comm. Math. Phys. 362 (2018), no. 2, 659-688, DOI 10.1007/s00220-018-3177-x. MR3843425

[41] P. Constantin, W. E, and E. S. Titi, Onsager's conjecture on the energy conservation for solutions of Euler's equation, Comm. Math. Phys. 165 (1994), no. 1, 207-209. MR.1298949

[42] P. Constantin and C. Foias, Navier-Stokes equations, Chicago Lectures in Mathematics, University of Chicago Press, Chicago, IL, 1988. MR972259

[43] P. Constantin, M. Ignatova, and H. Q. Nguyen, Inviscid limit for SQG in bounded domains, SIAM J. Math. Anal. 50 (2018), no. 6, 6196-6207, DOI 10.1137/18M1192743. MR.3882956

[44] P. Constantin and F. Ramos, Inviscid limit for damped and driven incompressible Navier-Stokes equations in $\mathbb{R}^{2}$, Comm. Math. Phys. 275 (2007), no. 2, 529-551, DOI 10.1007/s00220-007-0310-7. MR2335784

[45] P. Constantin, W. E, and E. S. Titi, Onsager's conjecture on the energy conservation for solutions of Euler's equation, Comm. Math. Phys. 165 (1994), no. 1, 207-209. MR.1298949

[46] D. Cordoba, D. Faraco, and F. Gancedo, Lack of uniqueness for weak solutions of the incompressible porous media equation, Arch. Ration. Mech. Anal. 200 (2011), no. 3, 725746, DOI 10.1007/s00205-010-0365-z. MR2796131

[47] G. Crippa, N. Gusev, S. Spirito, and E. Wiedemann, Non-uniqueness and prescribed energy for the continuity equation, Commun. Math. Sci. 13 (2015), no. 7, 1937-1947, DOI 10.4310/CMS.2015.v13.n7.a12. MR3393180

[48] M. Dai, Non-uniqueness of Leray-Hopf weak solutions of the $3 d$ Hall-MHD system, arXiv:1812.11311 (2018).

[49] M. Dai and H. Liu, Anomalous dissipation of energy and magnetic helicity for the electronmhd system, arXiv:1911.03953 (2019).

[50] V. Dallas and A. Alexakis, The signature of initial conditions on magnetohydrodynamic turbulence, The Astrophysical Journal Letters 788 (2014), no. 2, L36. 
[51] S. Daneri and L. Székelyhidi Jr., Non-uniqueness and h-principle for Hölder-continuous weak solutions of the Euler equations, Arch. Ration. Mech. Anal. 224 (2017), no. 2, 471514, DOI 10.1007/s00205-017-1081-8. MR3614753

[52] P. A. Davidson, An introduction to magnetohydrodynamics, Cambridge Texts in Applied Mathematics, Cambridge University Press, Cambridge, 2001. MR1825486

[53] P. A. Davidson, Y. Kaneda, K. Moffatt, and K. R. Sreenivasan (eds.), A voyage through turbulence, Cambridge University Press, Cambridge, 2011. MR2894536

[54] C. De Lellis and H. Kwon, On non-uniqueness of Hölder continuous globally dissipative Euler flows, arXiv preprint arXiv:2006.06482 (2020).

[55] C. De Lellis and L. Székelyhidi Jr., The Euler equations as a differential inclusion, Ann. of Math. (2) 170 (2009), no. 3, 1417-1436, DOI 10.4007/annals.2009.170.1417. MR2600877

[56] C. De Lellis and L. Székelyhidi Jr., On admissibility criteria for weak solutions of the Euler equations, Arch. Ration. Mech. Anal. 195 (2010), no. 1, 225-260, DOI 10.1007/s00205-0080201-x. MR2564474

[57] C. De Lellis and L. Székelyhidi Jr., The h-principle and the equations of fluid dynamics, Bull. Amer. Math. Soc. (N.S.) 49 (2012), no. 3, 347-375, DOI 10.1090/S0273-0979-2012-01376-9. MR.2917063

[58] C. De Lellis and L. Székelyhidi Jr., Dissipative continuous Euler flows, Invent. Math. 193 (2013), no. 2, 377-407, DOI 10.1007/s00222-012-0429-9. MR3090182

[59] C. De Lellis and L. Székelyhidi Jr., Dissipative Euler flows and Onsager's conjecture, J. Eur. Math. Soc. (JEMS) 16 (2014), no. 7, 1467-1505, DOI 10.4171/JEMS/466. MR.3254331

[60] C. De Lellis and L. Székelyhidi Jr., High dimensionality and h-principle in PDE, Bull. Amer. Math. Soc. (N.S.) 54 (2017), no. 2, 247-282, DOI 10.1090/bull/1549. MR3619726

[61] C. De Lellis and L. Székelyhidi Jr., On turbulence and geometry: from Nash to Onsager, Notices Amer. Math. Soc. 66 (2019), no. 5, 677-685. MR3929468

[62] L. De Rosa, Infinitely many Leray-Hopf solutions for the fractional Navier-Stokes equations, Comm. Partial Differential Equations 44 (2019), no. 4, 335-365, DOI 10.1080/03605302.2018.1547745. MR3941228

[63] L. De Rosa, On the helicity conservation for the incompressible Euler equations, Proc. Amer. Math. Soc. 148 (2020), no. 7, 2969-2979, DOI 10.1090/proc/14952. MR4099784

[64] R. J. DiPerna, Compensated compactness and general systems of conservation laws, Trans. Amer. Math. Soc. 292 (1985), no. 2, 383-420, DOI 10.2307/2000221. MR808729

[65] R. J. DiPerna and P.-L. Lions, Ordinary differential equations, transport theory and Sobolev spaces, Invent. Math. 98 (1989), no. 3, 511-547, DOI 10.1007/BF01393835. MR1022305

[66] R. J. DiPerna and A. J. Majda, Oscillations and concentrations in weak solutions of the incompressible fluid equations, Comm. Math. Phys. 108 (1987), no. 4, 667-689. MR877643

[67] C. R. Doering and J. D. Gibbon, Applied analysis of the Navier-Stokes equations, Cambridge Texts in Applied Mathematics, Cambridge University Press, Cambridge, 1995. MR 1325465

[68] J. Duchon and R. Robert, Inertial energy dissipation for weak solutions of incompressible Euler and Navier-Stokes equations, Nonlinearity 13 (2000), no. 1, 249-255, DOI 10.1088/0951-7715/13/1/312. MR.1734632

[69] G. Duvaut and J.-L. Lions, Inéquations en thermoélasticité et magnétohydrodynamique (French), Arch. Rational Mech. Anal. 46 (1972), 241-279, DOI 10.1007/BF00250512. MR.346289

[70] Y. Eliashberg and N. Mishachev, Introduction to the h-principle, Graduate Studies in Mathematics, vol. 48, American Mathematical Society, Providence, RI, 2002. MR.1909245

[71] A. Enciso, D. Peralta-Salas, and F. Torres de Lizaur, Helicity is the only integral invariant of volume-preserving transformations, Proc. Natl. Acad. Sci. USA 113 (2016), no. 8, 20352040, DOI 10.1073/pnas.1516213113. MR3474089

[72] L. Eskauriaza, G. A. Seregin, and V. Sverak, $L_{3, \infty}$-solutions of Navier-Stokes equations and backward uniqueness (Russian, with Russian summary), Uspekhi Mat. Nauk 58 (2003), no. 2(350), 3-44, DOI 10.1070/RM2003v058n02ABEH000609; English transl., Russian Math. Surveys 58 (2003), no. 2, 211-250. MR.1992563

[73] G. L. Eyink, Energy dissipation without viscosity in ideal hydrodynamics. I. Fourier analysis and local energy transfer, Phys. D 78 (1994), no. 3-4, 222-240, DOI 10.1016/01672789(94)90117-1. MR 1302409

[74] G. L. Eyink and K. R. Sreenivasan, Onsager and the theory of hydrodynamic turbulence, Rev. Modern Phys. 78 (2006), no. 1, 87-135, DOI 10.1103/RevModPhys.78.87. MR.2214822 
[75] E. B. Fabes, B. F. Jones, and N. M. Rivière, The initial value problem for the NavierStokes equations with data in $L^{p}$, Arch. Rational Mech. Anal. 45 (1972), 222-240, DOI 10.1007/BF00281533. MR 316915

[76] D. Faraco and S. Lindberg, Magnetic helicity and subsolutions in ideal mhd, arXiv:1801.04896 (2018).

[77] D. Faraco and S. Lindberg, Proof of Taylor's conjecture on magnetic helicity conservation, Comm. Math. Phys. 373 (2020), no. 2, 707-738, DOI 10.1007/s00220-019-03422-7. MR.4056647

[78] D. Faraco, S. Lindberg, and L. Székelyhidi, Jr., Bounded solutions of ideal MHD with compact support in space-time, arXiv preprint arXiv:1909.08678 (2019).

[79] C. Foiaş, Statistical study of Navier-Stokes equations. I, Rend. Sem. Mat. Univ. Padova 48 (1972), 219-348. MR 352733

[80] C. Foiaş, Statistical study of Navier-Stokes equations. II, Rend. Sem. Mat. Univ. Padova 49 (1973), 9-123. MR352733

[81] C. Foias, O. Manley, R. Rosa, and R. Temam, Navier-Stokes equations and turbulence, Encyclopedia of Mathematics and its Applications, vol. 83, Cambridge University Press, Cambridge, 2001. MR 1855030

[82] U. Frisch, Turbulence, Cambridge University Press, Cambridge, 1995. The legacy of A. N. Kolmogorov. MR.1428905

[83] H. Fujita and T. Kato, On the Navier-Stokes initial value problem. I, Arch. Rational Mech. Anal. 16 (1964), 269-315, DOI 10.1007/BF00276188. MR166499

[84] G. Furioli, P. G. Lemarié-Rieusset, and E. Terraneo, Unicité dans $L^{3}\left(\mathbb{R}^{3}\right)$ et d'autres espaces fonctionnels limites pour Navier-Stokes (French, with English and French summaries), Rev. Mat. Iberoamericana 16 (2000), no. 3, 605-667, DOI 10.4171/RMI/286. MR.1813331

[85] P. Germain, Multipliers, paramultipliers, and weak-strong uniqueness for the NavierStokes equations, J. Differential Equations 226 (2006), no. 2, 373-428, DOI 10.1016/j.jde.2005.10.007. MR.2237686

[86] M. L. Gromov, Convex integration of differential relations. I (Russian), Izv. Akad. Nauk SSSR Ser. Mat. 37 (1973), 329-343. MR0413206

[87] J. Guillod and V. Šverák, Numerical investigations of non-uniqueness for the Navier-Stokes initial value problem in borderline spaces, arXiv:1704.00560 (2017).

[88] M. Hofmanova, R. Zhu, and X. Zhu, Non-uniqueness in law of stochastic 3d Navier-Stokes equations, arXiv:1912.11841 (2019).

[89] E. Hopf, Über die Anfangswertaufgabe für die hydrodynamischen Grundgleichungen (German), Math. Nachr. 4 (1951), 213-231, DOI 10.1002/mana.3210040121. MR50423

[90] P. Isett, Hölder continuous Euler flows with compact support in time, ProQuest LLC, Ann Arbor, MI, 2013. Thesis (Ph.D.)-Princeton University. MR 3153420

[91] P. Isett, Nonuniqueness and existence of continuous, globally dissipative Euler flows, arXiv:1710.11186 (2017).

[92] P. Isett, On the endpoint regularity in Onsager's conjecture, arXiv:1706.01549 (2017).

[93] P. Isett, A proof of Onsager's conjecture, Ann. of Math. (2) 188 (2018), no. 3, 871-963, DOI 10.4007/annals.2018.188.3.4. MR3866888

[94] P. Isett and A. Ma, A direct approach to nonuniqueness and failure of compactness for the $S Q G$ equation, arXiv:2007.03078 (2020).

[95] P. Isett and S.-J. Oh, On nonperiodic Euler flows with Hölder regularity, Arch. Ration. Mech. Anal. 221 (2016), no. 2, 725-804, DOI 10.1007/s00205-016-0973-3. MR 3488536

[96] P. Isett and V. Vicol, Hölder continuous solutions of active scalar equations, Ann. PDE 1 (2015), no. 1, Art. 2, 77, DOI 10.1007/s40818-015-0002-0. MR3479065

[97] H. Jia and V. Šverák, Local-in-space estimates near initial time for weak solutions of the Navier-Stokes equations and forward self-similar solutions, Invent. Math. 196 (2014), no. 1, 233-265, DOI 10.1007/s00222-013-0468-x. MR.3179576

[98] H. Jia and V. Sverak, Are the incompressible $3 d$ Navier-Stokes equations locally illposed in the natural energy space?, J. Funct. Anal. 268 (2015), no. 12, 3734-3766, DOI 10.1016/j.jfa.2015.04.006. MR 3341963

[99] Y. Kaneda, T. Ishihara, M. Yokokawa, K. Itakura, and A. Uno, Energy dissipation rate and energy spectrum in high resolution direct numerical simulations of turbulence in a periodic box, Physics of Fluids 15 (2003), no. 2, L21-L24. 
[100] E. Kang and J. Lee, Remarks on the magnetic helicity and energy conservation for ideal magneto-hydrodynamics, Nonlinearity 20 (2007), no. 11, 2681-2689, DOI 10.1088/09517715/20/11/011. MR2361251

[101] B. Khesin, D. Peralta-Salas, and C. Yang, A basis of casimirs in $3 d$ magnetohydrodynamics, arXiv:1901.04404 (2019).

[102] B. Kirchheim, S. Müller, and V. Šverák, Studying nonlinear pde by geometry in matrix space, Geometric analysis and nonlinear partial differential equations, Springer, Berlin, 2003, pp. 347-395. MR2008346

[103] A. A. Kiselev and O. A. Ladyženskaya, On the existence and uniqueness of the solution of the nonstationary problem for a viscous, incompressible fluid (Russian), Izv. Akad. Nauk SSSR. Ser. Mat. 21 (1957), 655-680. MR0100448

[104] S. Klainerman, On Nash's unique contribution to analysis in just three of his papers, Bull. Amer. Math. Soc. (N.S.) 54 (2017), no. 2, 283-305, DOI 10.1090/bull/1560. MR3619727

[105] A. N. Kolmogoroff, Dissipation of energy in the locally isotropic turbulence, C. R. (Doklady) Acad. Sci. URSS (N.S.) 32 (1941), 16-18. MR0005851

[106] A. Kolmogoroff, The local structure of turbulence in incompressible viscous fluid for very large Reynold's numbers, C. R. (Doklady) Acad. Sci. URSS (N.S.) 30 (1941), 301-305. MR.0004146

[107] A. N. Kolmogoroff, On degeneration of isotropic turbulence in an incompressible viscous liquid, C. R. (Doklady) Acad. Sci. URSS (N. S.) 31 (1941), 538-540. MR0004568

[108] N. H. Kuiper, On $C^{1}$-isometric imbeddings. I, II, Nederl. Akad. Wetensch. Proc. Ser. A. 58 = Indag. Math. 17 (1955), 545-556, 683-689. MR0075640

[109] I. Kukavica, Pressure integrability conditions for uniqueness of mild solutions of the Navier-Stokes system, J. Differential Equations 223 (2006), no. 2, 427-441, DOI 10.1016/j.jde.2005.07.005. MR2214942

[110] I. Kukavica, Role of the pressure for validity of the energy equality for solutions of the Navier-Stokes equation, J. Dynam. Differential Equations 18 (2006), no. 2, 461-482, DOI 10.1007/s10884-006-9010-9. MR2229985

[111] I. Kukavica, The fractal dimension of the singular set for solutions of the Navier-Stokes system, Nonlinearity 22 (2009), no. 12, 2889-2900, DOI 10.1088/0951-7715/22/12/005. MR.2557452

[112] O. A. Ladyženskaja, Uniqueness and smoothness of generalized solutions of Navier-Stokes equations (Russian), Zap. Naučn. Sem. Leningrad. Otdel. Mat. Inst. Steklov. (LOMI) 5 (1967), 169-185. MR0236541

[113] P. G. Lemarié-Rieusset, Recent developments in the Navier-Stokes problem, Chapman \& Hall/CRC Research Notes in Mathematics, vol. 431, Chapman \& Hall/CRC, Boca Raton, FL, 2002. MR 1938147

[114] P. G. Lemarié-Rieusset, The Navier-Stokes problem in the 21st century, CRC Press, Boca Raton, FL, 2016. MR3469428

[115] J. Leray, Sur le mouvement d'un liquide visqueux emplissant l'espace (French), Acta Math. 63 (1934), no. 1, 193-248, DOI 10.1007/BF02547354. MR 1555394

[116] T. M. Leslie and R. Shvydkoy, Conditions implying energy equality for weak solutions of the Navier-Stokes equations, SIAM J. Math. Anal. 50 (2018), no. 1, 870-890, DOI 10.1137/16M1104147. MR3759872

[117] J.-L. Lions, Quelques résultats d'existence dans des équations aux dérivées partielles non linéaires (French), Bull. Soc. Math. France 87 (1959), 245-273. MR115019

[118] J. L. Lions, Sur la régularité et l'unicité des solutions turbulentes des équations de Navier Stokes (French), Rend. Sem. Mat. Univ. Padova 30 (1960), 16-23. MR115018

[119] P.-L. Lions, Mathematical topics in fluid mechanics. Vol. 2, Oxford Lecture Series in Mathematics and its Applications, vol. 10, The Clarendon Press, Oxford University Press, New York, 1998. Compressible models; Oxford Science Publications. MR.1637634

[120] P.-L. Lions and N. Masmoudi, Uniqueness of mild solutions of the Navier-Stokes system in $L^{N}$, Comm. Partial Differential Equations 26 (2001), no. 11-12, 2211-2226, DOI 10.1081/PDE-100107819. MR.1876415

[121] T. Luo and P. Qu, Non-uniqueness of weak solutions to 2D hypoviscous Navier-Stokes equations, J. Differential Equations 269 (2020), no. 4, 2896-2919, DOI 10.1016/j.jde.2020.02.014. $\operatorname{MR} 4097236$ 
[122] T. Luo and E. S. Titi, Non-uniqueness of weak solutions to hyperviscous Navier-Stokes equations: on sharpness of J.-L. Lions exponent, Calc. Var. Partial Differential Equations 59 (2020), no. 3, Paper No. 92, 15, DOI 10.1007/s00526-020-01742-4. MR 4092687

[123] X. Luo, Stationary solutions and nonuniqueness of weak solutions for the Navier-Stokes equations in high dimensions, Arch. Ration. Mech. Anal. 233 (2019), no. 2, 701-747, DOI 10.1007/s00205-019-01366-9. MR.3951691

[124] A. J. Majda and A. L. Bertozzi, Vorticity and incompressible flow, Cambridge Texts in Applied Mathematics, vol. 27, Cambridge University Press, Cambridge, 2002. MR.1867882

[125] C. Marchioro and M. Pulvirenti, Mathematical theory of incompressible nonviscous fluids, Applied Mathematical Sciences, vol. 96, Springer-Verlag, New York, 1994. MR 1245492

[126] C. Meneveau and K. R. Sreenivasan, The multifractal nature of turbulent energy dissipation, J. Fluid Mech. 224 (1991), 429-484.

[127] P. D. Mininni and A. Pouquet, Finite dissipation and intermittency in magnetohydrodynamics, Physical Review E 80 (2009), no. 2, 025401.

[128] S. Modena and G. Sattig, Convex integration solutions to the transport equation with full dimensional concentration, Ann. Inst. H. Poincaré Anal. Non Linéaire 37 (2020), no. 5, 1075-1108, DOI 10.1016/j.anihpc.2020.03.002. MR4138227

[129] S. Modena and L. Székelyhidi Jr., Non-uniqueness for the transport equation with Sobolev vector fields, Ann. PDE 4 (2018), no. 2, Paper No. 18, 38, DOI 10.1007/s40818-018-0056-x. MR 3884855

[130] S. Modena and L. Székelyhidi Jr., Non-renormalized solutions to the continuity equation, Calculus of Variations and Partial Differential Equations 58 (2019), no. 6, 208.

[131] H. K. Moffatt, The degree of knottedness of tangled vortex lines, J. Fluid Mech. 35 (1969), no. 1, 117-129.

[132] H. K. Moffatt, Magnetic relaxation and the Taylor conjecture, Journal of Plasma Physics 81 (2015), no. 6.

[133] A. S. Monin and A. M. Yaglom, Statistical fluid mechanics: mechanics of turbulence. Vol. II, Dover Publications, Inc., Mineola, NY, 2007. Translated from the 1965 Russian original; Edited and with a preface by John L. Lumley; English edition updated, augmented and revised by the authors; Reprinted from the 1975 edition. MR 2406668

[134] S. Müller and V. Šverák, Convex integration for Lipschitz mappings and counterexamples to regularity, Ann. of Math. (2) 157 (2003), no. 3, 715-742, DOI 10.4007/annals.2003.157.715. MR.1983780

[135] J. Nash, $C^{1}$ isometric imbeddings, Ann. of Math. (2) 60 (1954), 383-396, DOI 10.2307/1969840. MR65993

[136] F. Nguyen, J.-P. Laval, P. Kestener, A. Cheskidov, R. Shvydkoy, and B. Dubrulle, Local estimates of Hölder exponents in turbulent vector fields, Physical Review E 99 (2019), no. 5, 053114.

[137] M. Novack, Nonuniqueness of weak solutions to the 3 dimensional quasi-geostrophic equations, SIAM J. Math. Anal. 52 (2020), no. 4, 3301-3349, DOI 10.1137/19M1281009. MR 4126319

[138] L. Onsager, Statistical hydrodynamics, Nuovo Cimento (9) 6 (1949), no. Suppl, Supplemento, no. 2 (Convegno Internazionale di Meccanica Statistica), 279-287. MR36116

[139] S. Ortolani and D. Schnack, Magnetohydrodynamics of plasma relaxation, World Scientific, 1993.

[140] W. S. Ożański, The partial regularity theory of Caffarelli, Kohn, and Nirenberg and its sharpness, Lecture Notes in Mathematical Fluid Mechanics, Birkhäuser/Springer, Cham, 2019. MR3969978

[141] B. R. Pearson, P.-A. Krogstad, and W. Van De Water, Measurements of the turbulent energy dissipation rate, Physics of Fluids 14 (2002), no. 3, 1288-1290.

[142] G. Prodi, Un teorema di unicità per le equazioni di Navier-Stokes (Italian), Ann. Mat. Pura Appl. (4) 48 (1959), 173-182, DOI 10.1007/BF02410664. MR126088

[143] J. C. Robinson, J. L. Rodrigo, and W. Sadowski, The three-dimensional Navier-Stokes equations, Cambridge Studies in Advanced Mathematics, vol. 157, Cambridge University Press, Cambridge, 2016. Classical theory. MR3616490

[144] J. C. Robinson and W. Sadowski, Decay of weak solutions and the singular set of the three-dimensional Navier-Stokes equations, Nonlinearity 20 (2007), no. 5, 1185-1191, DOI 10.1088/0951-7715/20/5/007. MR2312388 
[145] V. Scheffer, Partial regularity of solutions to the Navier-Stokes equations, Pacific J. Math. 66 (1976), no. 2, 535-552. MR454426

[146] V. Scheffer, Hausdorff measure and the Navier-Stokes equations, Comm. Math. Phys. 55 (1977), no. 2, 97-112. MR510154

[147] V. Scheffer, An inviscid flow with compact support in space-time, J. Geom. Anal. 3 (1993), no. 4, 343-401, DOI 10.1007/BF02921318. MR1231007

[148] M. Sermange and R. Temam, Some mathematical questions related to the MHD equations, Comm. Pure Appl. Math. 36 (1983), no. 5, 635-664, DOI 10.1002/cpa.3160360506. $\operatorname{MR} 716200$

[149] J. Serrin, On the interior regularity of weak solutions of the Navier-Stokes equations, Arch. Rational Mech. Anal. 9 (1962), 187-195, DOI 10.1007/BF00253344. MR136885

[150] J. Serrin, The initial value problem for the Navier-Stokes equations, Nonlinear Problems (Proc. Sympos., Madison, Wis., 1962), Univ. of Wisconsin Press, Madison, Wis., 1963, pp. 69-98. MR0150444

[151] M. Shinbrot, The energy equation for the Navier-Stokes system, SIAM J. Math. Anal. 5 (1974), 948-954, DOI 10.1137/0505092. MR435629

[152] A. Shnirelman, Weak solutions with decreasing energy of incompressible Euler equations, Comm. Math. Phys. 210 (2000), no. 3, 541-603, DOI 10.1007/s002200050791. MR1777341

[153] A. Shnirelman, On the nonuniqueness of weak solution of the Euler equation, Comm. Pure Appl. Math. 50 (1997), no. 12, 1261-1286, DOI 10.1002/(SICI)10970312(199712)50:12〈1261::AID-CPA3〉3.3.CO;2-4. MR.1476315

[154] R. Shvydkoy, Lectures on the Onsager conjecture, Discrete Contin. Dyn. Syst. Ser. S 3 (2010), no. 3, 473-496, DOI 10.3934/dcdss.2010.3.473. MR2660721

[155] R. Shvydkoy, Convex integration for a class of active scalar equations, J. Amer. Math. Soc. 24 (2011), no. 4, 1159-1174, DOI 10.1090/S0894-0347-2011-00705-4. MR2813340

[156] R. Shvydkoy, Homogeneous solutions to the $3 D$ Euler system, Trans. Amer. Math. Soc. 370 (2018), no. 4, 2517-2535, DOI 10.1090/tran/7022. MR3748575

[157] K. R. Sreenivasan, On the fine-scale intermittency of turbulence, Journal of Fluid Mechanics 151 (1985), 81-103.

[158] K. R. Sreenivasan, An update on the energy dissipation rate in isotropic turbulence, Phys. Fluids 10 (1998), no. 2, 528-529, DOI 10.1063/1.869575. MR.1604680

[159] K. R. Sreenivasan, S. I. Vainshtein, R. Bhiladvala, I. San Gil, S. Chen, and N. Cao, Asymmetry of Velocity Increments in Fully Developed Turbulence and the Scaling of Low-Order Moments, Phys. Rev. Lett. 77 (1996), no. 8, 1488-1491.

[160] P.-L. Sulem and U. Frisch, Bounds on energy flux for finite energy turbulence, J. Fluid Mech. 72 (1975), no. 3, 417-423.

[161] L. Székelyhidi, Weak solutions to the incompressible Euler equations with vortex sheet initial data (English, with English and French summaries), C. R. Math. Acad. Sci. Paris 349 (2011), no. 19-20, 1063-1066, DOI 10.1016/j.crma.2011.09.009. MR.2842999

[162] L. Székelyhidi Jr., From isometric embeddings to turbulence, HCDTE Lecture Notes. Part II. Nonlinear hyperbolic PDEs, dispersive and transport equations, AIMS Ser. Appl. Math., vol. 7, Am. Inst. Math. Sci. (AIMS), Springfield, MO, 2013, pp. 63. MR3340997

[163] L. Székelyhidi Jr., Relaxation of the incompressible porous media equation (English, with English and French summaries), Ann. Sci. Éc. Norm. Supér. (4) 45 (2012), no. 3, 491-509, DOI 10.24033/asens.2171. MR3014484

[164] T. Tao, 255b, Notes 2: Onsager's conjecture.

[165] L. Tartar, Compensated compactness and applications to partial differential equations, Nonlinear analysis and mechanics: Heriot-Watt Symposium, Vol. IV, Res. Notes in Math., vol. 39, Pitman, Boston, Mass.-London, 1979, pp. 136-212. MR.584398

[166] L. Tartar, The compensated compactness method applied to systems of conservation laws, Systems of nonlinear partial differential equations (Oxford, 1982), NATO Adv. Sci. Inst. Ser. C Math. Phys. Sci., vol. 111, Reidel, Dordrecht, 1983, pp. 263-285. MR725524

[167] L. Tartar, The compensated compactness method applied to systems of conservation laws, Systems of nonlinear partial differential equations (Oxford, 1982), NATO Adv. Sci. Inst. Ser. C Math. Phys. Sci., vol. 111, Reidel, Dordrecht, 1983, pp. 263-285. MR725524

[168] J. B. Taylor, Relaxation of toroidal plasma and generation of reverse magnetic fields, Physical Review Letters 33 (1974), no. 19, 1139. 
[169] J. B. Taylor, Relaxation and magnetic reconnection in plasmas, Reviews of Modern Physics 58 (1986), no. 3, 741.

[170] R. Temam, Navier-Stokes equations and nonlinear functional analysis, 2nd ed., CBMSNSF Regional Conference Series in Applied Mathematics, vol. 66, Society for Industrial and Applied Mathematics (SIAM), Philadelphia, PA, 1995. MR 1318914

[171] T.-P. Tsai, Lectures on Navier-Stokes equations, Graduate Studies in Mathematics, vol. 192, American Mathematical Society, Providence, RI, 2018. MR3822765

[172] J. C. Vassilicos, Dissipation in turbulent flows, Annual review of fluid mechanics. Vol. 47, Annu. Rev. Fluid Mech., vol. 47, Annual Reviews, Palo Alto, CA, 2015, pp. 95-114. MR.3727163

[173] S. M. Vishik and F. V. Dolzhanskii, Analogs of the euler-lagrange equations and magnetohydrodynamics equations related to lie groups, Sov. Math. Doklady, vol. 19, 1978, pp. 149-153.

[174] E. Wiedemann, Existence of weak solutions for the incompressible Euler equations, Ann. Inst. H. Poincaré Anal. Non Linéaire 28 (2011), no. 5, 727-730, DOI 10.1016/j.anihpc.2011.05.002. MR2838398

[175] E. Wiedemann, Inviscid symmetry breaking with non-increasing energy (English, with English and French summaries), C. R. Math. Acad. Sci. Paris 351 (2013), no. 23-24, 907-910, DOI 10.1016/j.crma.2013.10.021. MR3133602

[176] E. Wiedemann, Weak-strong uniqueness in fluid dynamics, Partial differential equations in fluid mechanics, London Math. Soc. Lecture Note Ser., vol. 452, Cambridge Univ. Press, Cambridge, 2018, pp. 289-326. MR 3838055

[177] L. Woltjer, On hydromagnetic equilibrium, Proc. Nat. Acad. Sci. U.S.A. 44 (1958), 833-841, DOI 10.1073/pnas.44.9.833. MR.96485

[178] L. Woltjer, A theorem on force-free magnetic fields, Proc. Nat. Acad. Sci. U.S.A. 44 (1958), no. $6,489$.

[179] K. Yamazaki, Remarks on the non-uniqueness in law of the Navier-Stokes equations up to the J.-L. Lions' exponent, arXiv:2006.11861 (2020).

Department of Mathematics, Princeton University, Princeton, New Jersey

Email address: buckmaster@math.princeton.edu

Courant Institute for Mathematical Sciences, New York University, New York, NEW YORK

Email address: vicol@cims.nyu.edu 